\title{
TRIM9 short isoform preferentially promotes DNA and RNA virus-induced production of type I interferon by recruiting GSK3ß to TBK1
}

\author{
Yunfei Qin ${ }^{1, *}$, Qingxiang Liu ${ }^{1, *}$, Shuo Tian ${ }^{1, *}$, Weihong Xie' ${ }^{2}$ Jun Cui ${ }^{1,3}$, Rong-Fu Wang ${ }^{4-6}$ \\ ${ }^{1}$ Key Laboratory of Gene Engineering of the Ministry of Education, State Key Laboratory of Biocontrol, School of Life Scienc- \\ es, ${ }^{2}$ Zhongshan School of Medicine, ${ }^{3}$ Collaborative Innovation Center of Cancer Medicine, Sun Yat-sen University, Guangzhou, \\ Guangdong, China; ${ }^{4}$ Houston Methodist Research Institute, Houston, TX 77030, USA; ${ }^{5}$ Department of Microbiology and Immu- \\ nology, Weill Cornell Medical College, Cornell University, New York, NY 10065, USA; ${ }^{6}$ Institute of Biosciences and Technology, \\ College of Medicine, Texas A \& M University, Houston, TX 77030, USA
}

Type I interferon (IFN) is an important component of antiviral innate immune signaling mediated by viral DNA and RNA recognition by the DNA sensor cGAS and RNA sensors RIG-I and MDA5. Activation of these DNA and RNA sensors leads to the recruitment of STING and MAVS, respectively, and converges on TANK-binding kinase 1 (TBK1) signaling for subsequent phosphorylation of IFN regulatory factor 3 (IRF3). However, the mechanisms that control TBK1 activation are still poorly defined. Here, we identify tripartite motif 9 short isoform (TRIM9s) as a positive regulator in type I IFN signaling. Upon viral infection, TRIM9s undergoes Lys-63-linked auto-polyubiquitination and serves as a platform to bridge GSK3 $\beta$ to TBK1, leading to the activation of IRF3 signaling. Interestingly, we found that TRIM9s selectively inhibits the production of pro-inflammatory cytokines, but enhances the expression of type I IFNs as well as IFN-stimulated genes, in response to viral infection. Our findings reveal novel dual functions of TRIM9s in antiviral immunity, which serve to balance pro-inflammatory response and production of type I IFNs.

Keywords: TRIM9; GSK3 $\beta$; TBK1; ubiquitination

Cell Research (2016) 26:613-628. doi:10.1038/cr.2016.27; published online 26 February 2016

\section{Introduction}

An effective immune response against RNA and DNA viruses depends on the induction of type I interferons (IFNs), such as IFN $\alpha / \beta$, upon the recognition of virus-specific pathogen-associated molecular patterns (PAMPs) [1, 2]. Viral RNA and DNA are mainly recognized by a variety of pattern recognition receptors, such as Toll-like receptors (TLRs), retinoic acid-inducible gene-I (RIG-I)-like receptors (RLRs), NOD-like receptors (NLRs) and several DNA sensors [3-6]. While many TLRs and NLRs including TLR3, TLR7/9 and Nod2

\footnotetext{
*These three authors contributed equally to this work.

Correspondence: Rong-Fu Wang ${ }^{\mathrm{a}}$, Jun Cui ${ }^{\mathrm{b}}$

aE-mail: rwang3@tmhs.org

bE-mail: cuij5@mail.sysu.edu.cn

Received 11 June 2015; revised 16 October 2015; accepted 2 December 2015; published online 26 February 2016
}

initiate type I IFN production upon recognition of viral dsRNAs or ssRNAs [2], RLRs including RIG-I, MDA5 and LGP2 are the primary sensors of intracellular viral RNAs [4-7]. Upon recognition of viral RNAs, RLRs mediate the recruitment of the mitochondrial adaptor protein MAVS to activate the IRF3/IRF7 and NF- $\kappa B$ signaling pathways [8]. Recently, a number of viral DNA sensors, including DDX41, IFI16 and cGAS, have been reported to initiate type I IFN production through another adaptor protein, STING [9-11]. Both MAVS- and STING-dependent signaling cascades result in the recruitment and activation of TANK-binding kinase 1 (TBK1), and subsequently activation of transcription factors IRF3/7 and NF- $\mathrm{B}[11,12]$.

The activity of TBK1 is regulated by multiple posttranslational modifications. TBK1 undergoes robust ubiquitination with Lys (K) 63 linkage induced by mind bomb and Ndrp1 in response to RLR and TLR ligands, respectively, to promote type I IFN production $[13,14]$. 
On the other hand, K48-linked ubiquitination of TBK1, mediated by NLRP4/DTX4 or TRIP $[15,16]$, results in the degradation of TBK1, thus inhibiting the TBK1IRF3 signaling cascade. In addition, several regulators, including SHIP1, PPM1B and glucocorticoids, have been reported to modulate TBK1 activity through regulating TBK1 phosphorylation [17]. Recently, GSK3 $\beta$ was shown to interact with TBK1 during virus infection and enhance TBK1 oligomerization and auto-phosphorylation at Ser172 [18]. Although much progress has been made in understanding the regulation of TBK1, mechanistic details regarding TBK1 activation still need to be elucidated in order to better understand virus-induced immune responses.

The tripartite motif protein (TRIM) family contains over 70 members in humans. Most TRIM family members share a similar structure of a really interesting new gene (RING) domain and are believed to function as E3 ubiquitin ligases [19]. Accumulating evidence suggests that many TRIM proteins are involved in antiviral immunity. For example, TRIM4 and TRIM25 regulate the ubiquitination of RIG-I to promote type I IFN production and NF$\kappa \mathrm{B}$ activity [20, 21]. Furthermore, TRIM32 and TRIM56 positively regulate DNA virus-induced type I IFN signaling by interacting with STING and targeting it for K63linked ubiquitination $[22,23]$. On the other hand, TRIM21 negatively regulates the innate immune response to intracellular dsDNA by targeting DDX41 for degradation [24]. A recent systematic analysis of 75 human TRIM proteins, based on the screening of the complementary DNA clone library, indicated that nearly half of TRIM proteins serve as positive regulators of antiviral responses [25]. However, the detailed mechanisms by which TRIM proteins affect type I IFN signaling remain largely unknown.

There are two TRIM9 (also known as SPRING) isoforms, TRIM9 isoform 1 (long form; TRIM9l) and TRIM9 isoform 2 (short form; TRIM9s) in human cells [26]. TRIM9 is highly expressed in the central nervous system [26, 27]. Recently, TRIM91 was reported to negatively regulate NF- $\mathrm{KB}$ signaling by preventing $\beta$-TrCP binding to its substrate I $\mathrm{KB}$ [28]. However, the role of TRIM9 in virus-induced type I IFN signaling and inflammation is still not clear. In this study, we demonstrate that TRIM9s, but not TRIM91, is a positive regulator of type I IFN signaling in response to both DNA and RNA viruses. Upon viral infection, TRIM9s undergoes auto-regulated K63-linked polyubiquitination and recruits GSK3 $\beta$ to TBK1 to initiate the oligomerization of TBK1. Interestingly, TRIM9s deficiency significantly abrogates the production of type I IFNs but enhances NF- $\kappa B$-mediated pro-inflammatory cytokine production. Our findings demonstrate that TRIM9s acts as a mediator of antiviral immunity by balancing IRF3 and NF- $\mathrm{KB}$ signaling.

\section{Results}

\section{Identification of TRIM9s as a novel antiviral protein}

To investigate the roles of TRIM family proteins in antiviral immunity, we screened a panel of TRIM proteins using a vesicular stomatitis virus (VSV) infection assay. We transfected 293T cells with individual TRIM plasmids and infected them with VSV tagged with enhanced green fluorescent protein (VSV-eGFP). The percentage of infected (GFP-positive) cells was determined by flow cytometry (Figure 1A). Notably, TRIM8, TRIM14, TRIM21, TRIM11 and TRIM38 that were previously reported to positively or negatively modulate innate immune responses were identified as hits in the screening (Figure 1B), thus validating this experimental approach [25, 29-33]. These data also led to the identification of TRIM9s (short isoform of TRIM9) as a potent antiviral protein against VSV infection (Figure 1B). Since TRIM9 has two isoforms according to the NCBI database, we cloned both TRIM9 isoforms from human peripheral blood mononuclear cells (PBMCs) (Figure $1 \mathrm{C}$ ), and used them to test the specificity of two commercial TRIM9 antibodies. We found that the antibody from Proteintech (10786-1-AP, -TRIM9) can recognize both TRIM9 isoforms, whereas the antibody from Origene (TA800044, $\alpha$-TRIM9s) specifically recognizes TRIM9s (Supplementary information, Figure S1A). Using fluorescence microscopy and flow cytometry, we found that overexpression of TRIM9s decreased the number of GFP-positive cells compared with TRIM91 or empty vector (Figure 1D and 1E). These data suggest that TRIM9s facilitates cellular resistance to viral infection. TRIM91 was reported to activate type I IFN signaling by Versteeg et al. [25]. In order to confirm the function of TRIM91, we overexpressed TRIM91 at different doses and found that TRIM91 had moderate antiviral activity only when it was highly expressed (Figure 1F), which is consistent with the previous report [25].

TRIM9 has been previously reported to be specifically expressed in brain [26, 27]. However, TRIM9 can also be detected in human monocyte-derived macrophages and peripheral blood lymphocytes [34]. To investigate the expression pattern of TRIM9 isoforms, we performed quantitative PCR and immunoblot analysis to detect TRIM9s and TRIM91 at both mRNA and protein levels in several human tissues and cells. The results showed that TRIM9s and TRIM91 are widely expressed in liver, spleen, lung, PBMCs and several common cell lines, such as U251, A549 and 293T cells. The mRNA and protein levels of TRIM9s and TRIM9l are similar in the different human tissues and cell 
A

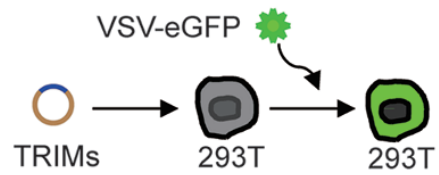

C<smiles>C1CCC2(CC1)CCCC2</smiles>

\begin{tabular}{lllllllll}
\hline & TRIM9I & IING & BB & BB & CC & COS & FN3 & SPRYIPRY \\
\hline
\end{tabular} TRIM9s RING | BB BB | CC 1 COS

Flag-TRIM9

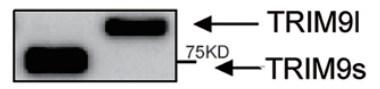

D $\quad \beta$-actin

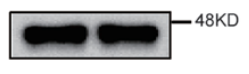

E
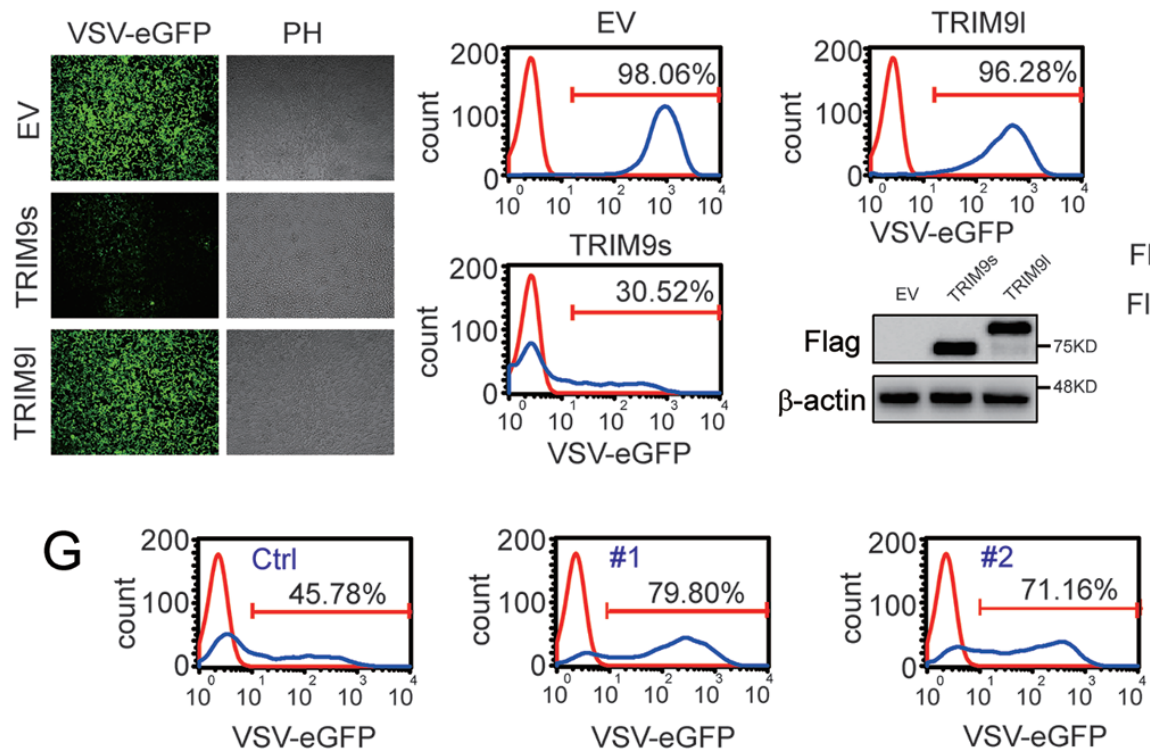

$\mathrm{H}$

Ctrl siRNA +---

TRIM9I SIRNA - 1\# 2\#- -

TRIM9s SIRNA - $-\quad-1 \# 2 \#$

TRIM9

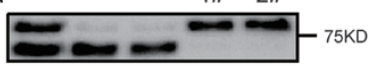

$\beta$-actin
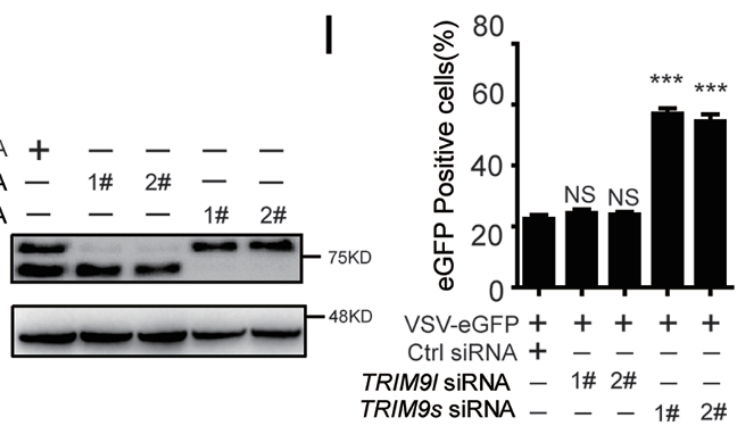

TRIM9s SIRNA - - - $\# 2 \#$

L

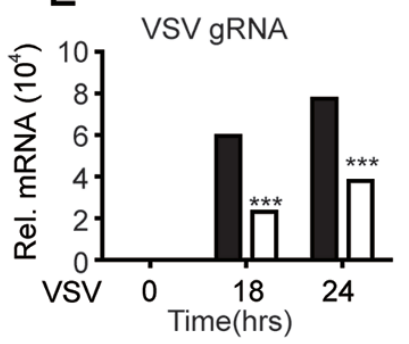

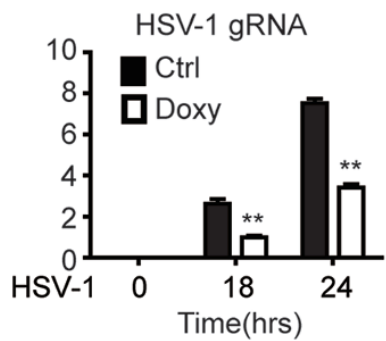

$\mathrm{M}$

$J$

\section{Flag-TRIM9I - \\ Flag-TRIM9I 0200400800 ng}
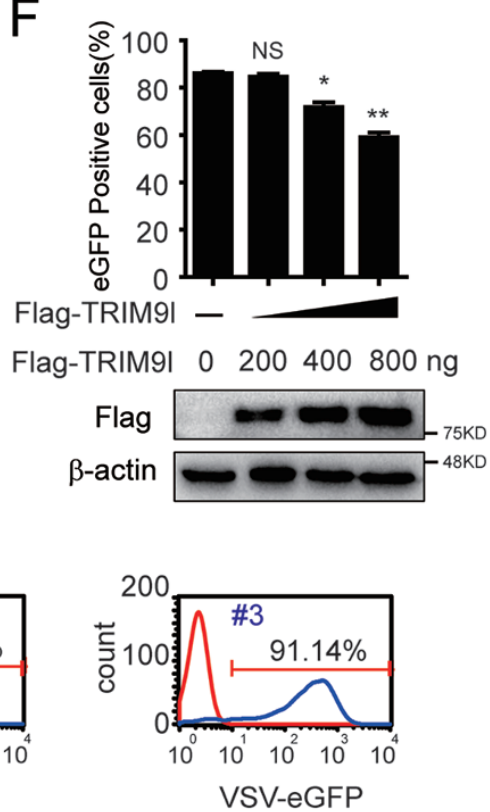

- Empty vector

v RIG-I (CARD)

- TRIMs

VSV-eGFP

WT 9s KO 
lines examined (Supplementary information, Figure S1BS1C). These results suggest that the expression of TRIM9 (short and long isoforms) is not restricted to brain.

To confirm the antiviral function of TRIM9 under physiological conditions, we selected three specific small interfering RNAs (siRNAs) to knock down the expression of TRIM9. All three TRIM9 siRNAs efficiently inhibited endogenous TRIM9s and TRIM91 expression in A549 cells (Supplementary information, Figure S2A). Specific knockdown of TRIM9 resulted in an increased number of GFP-positive cells, suggesting enhanced viral infection (Figure 1G). To better understand the function of the TRIM9 isoforms in antiviral immunity, we designed specific siRNAs to knockdown the short or long isoform of TRIM9 (Figure 1H). We found that knockdown of TRIM9s, but not TRIM9l, resulted in more GFP-positive cells (Figure 1I). Furthermore, we generated TRIM9s-knockout (KO) cells using the CRISPR/Cas9 technology (Figure 1J and Supplementary information, Figure S2B) [35], and observed considerably increased GFP signals in TRIM9s KO cells after VSV-eGFP infection (Figure 1K). All these data suggest that TRIM9s is a critical antiviral protein in response to viral infection.

To further explore the roles of TRIM9s in response to both DNA and RNA virus infection and viral replication, we established a doxycycline-inducible TRIM9s-overexpressing cell line (Supplementary information, Figure S2C). We observed lower levels of viral glycoprotein mRNAs (gRNA) within cells upon doxycycline treatment after VSV or herpes simplex virus 1 (HSV-1) infection (Figure 1L). Consistently, we found higher levels of viral gRNA in TRIM9s-KO cells compared with wild-type (WT) cells (Figure 1M). Taken together, these results suggest that TRIM9s is a potent, protective antiviral protein, inhibiting the replication of both RNA and DNA viruses.

\section{TRIM9s enhances IRF3 signaling through promoting TBK1 phosphorylation}

Next, we investigated whether TRIM9s protects cells from viral infection by enhancing type I IFN signaling. We found that TRIM9s promoted IFN $\beta$ expression in cells infected with VSV (Figure 2A). Furthermore, we tested the effect of TRIM9 on the phosphorylation of the transcription factor IRF3. As shown in Figure 2B, the phosphorylation of IRF3 (p-IRF3) in TRIM9s-overexpressing cells was at least two-fold higher than that in TRIM91-overexpressing or control cells after VSV infection. Collectively, these results suggest that TRIM9s enhances the antiviral immunity by inducing phosphorylation of IRF3 after viral infection.

To determine the molecular mechanisms by which TRIM9s positively regulates type I IFN signaling, we co-transfected $293 \mathrm{~T}$ cells with expression vectors encoding RIG-I (2CARD), MDA5, cGAS/STING, MAVS, TBK1, IKKi or IRF3 (5D) (a constitutively active mutant of IRF3) together with increasing amounts of TRIM9s plus the IFN-stimulated response element (ISRE) luciferase reporter. We found that TRIM9s enhanced ISRE reporter activity induced by RIG-I (2CARD), MDA5, cGAS/STING, MAVS and TBK1, but not IKKi or IRF3 (5D) (Figure 2C and Supplementary information, Figure S3A). Conversely, TRIM91 did not enhance ISRE reporter activity (Supplementary information, Figure S3B). Moreover, knockdown of TRIM9 with siRNAs impaired

Figure 1 TRIM9s is an antiviral protein. (A) Schematic overview of the VSV-eGFP infection assay. (B) 293T cells were transfected with plasmids of 29 TRIMs, pcDNA3.1 empty vector and RIG-I (2CARD) for $24 \mathrm{~h}$. The cells were infected with VSV-eG$\mathrm{FP}$ at an MOI of 0.01 for $24 \mathrm{~h}$, and the GFP-positive cells were determined by flow cytometry. (C) Immunoblot (IB) analysis (lower) of 293T cells transfected with two isoforms of TRIM9 (upper). TRIM9s, TRIM9 short isoform; TRIM9I, TRIM9 long isoform. (D, E) 293T cells were transfected with pcDNA3.1 empty vector, TRIM9s or TRIM9I (200 ng), and then infected with VSV-eGFP. Viral infection was analyzed by fluorescence microscopy (with phase contrast as a control) (D) and flow cytometry $(E)$, and the expression of two isoforms was analyzed by IB analysis (bottom of E). (F) 293T cells were transfected with the empty vector (no wedge) or increasing amounts (wedge, 200, 400, $800 \mathrm{ng}$ ) of expression vector for TRIM9I for 24h, and then infected with VSV-eGFP at an MOI of 0.01 for $18 \mathrm{~h}$. Viral infection was analyzed by flow cytometry, and the expression of TRIM9I was analyzed by IB analysis (bottom of F). (G) FACS analysis of A549 cells transfected with control siRNA (Ctrl) or TRIM9 siRNAs, and then infected with VSV-eGFP at an MOI of 0.01 for $24 \mathrm{~h}$. (H) The efficiency of knockdown of TRIM9s or TRIM9I in A549 cells with specific siRNAs. (I) A549 cells were transfected with siRNAs of TRIM9s, TRIM9I or control for $24 \mathrm{~h}$, and then infected with VSV-eGFP at an MOI of 0.01 for $18 \mathrm{~h}$. Viral replication was analyzed by FACS. (J) IB analysis of endogenous TRIM9 in TRIM9s knockout (9s KO) and wild-type (WT) A549 cells. $\beta$-actin serves as a loading control. (K) Flow cytometry assessing the infection in WT or 9s KO A549 cells infected with VSV-eGFP at an MOI of 0.01. Numbers above the bracketed lines indicate the percentage of cells expressing eGFP (infected cells). (L) Real-time PCR analysis of VSV or HSV$1 \mathrm{gRNA}$ in A549 cells treated with doxycycline (Doxy; $100 \mathrm{ng} / \mathrm{ml}$ ) or DMEM at the indicated time. (M) Real-time PCR analysis of VSV or HSV-1 gRNA in WT or 9s KO A549 cells at the indicated time points. ${ }^{*} P<0.05,{ }^{* *} P<0.01$ and ${ }^{* * *} P<0.001$, versus WT cells with the same treatment (Student's $t$-test). Data are representative of three independent experiments (mean \pm SD in $\mathbf{B}$, $\mathbf{F}, \mathbf{I}, \mathbf{L}$ and $\mathbf{M}$ ). 


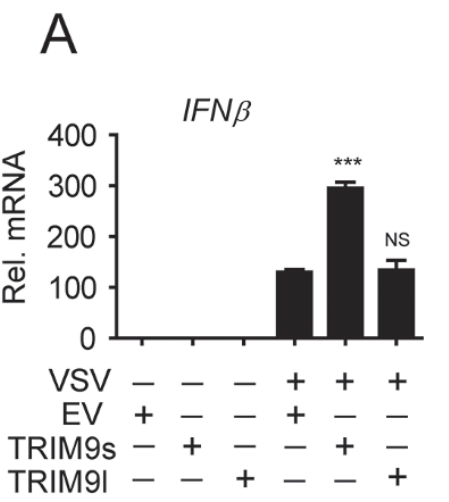

B
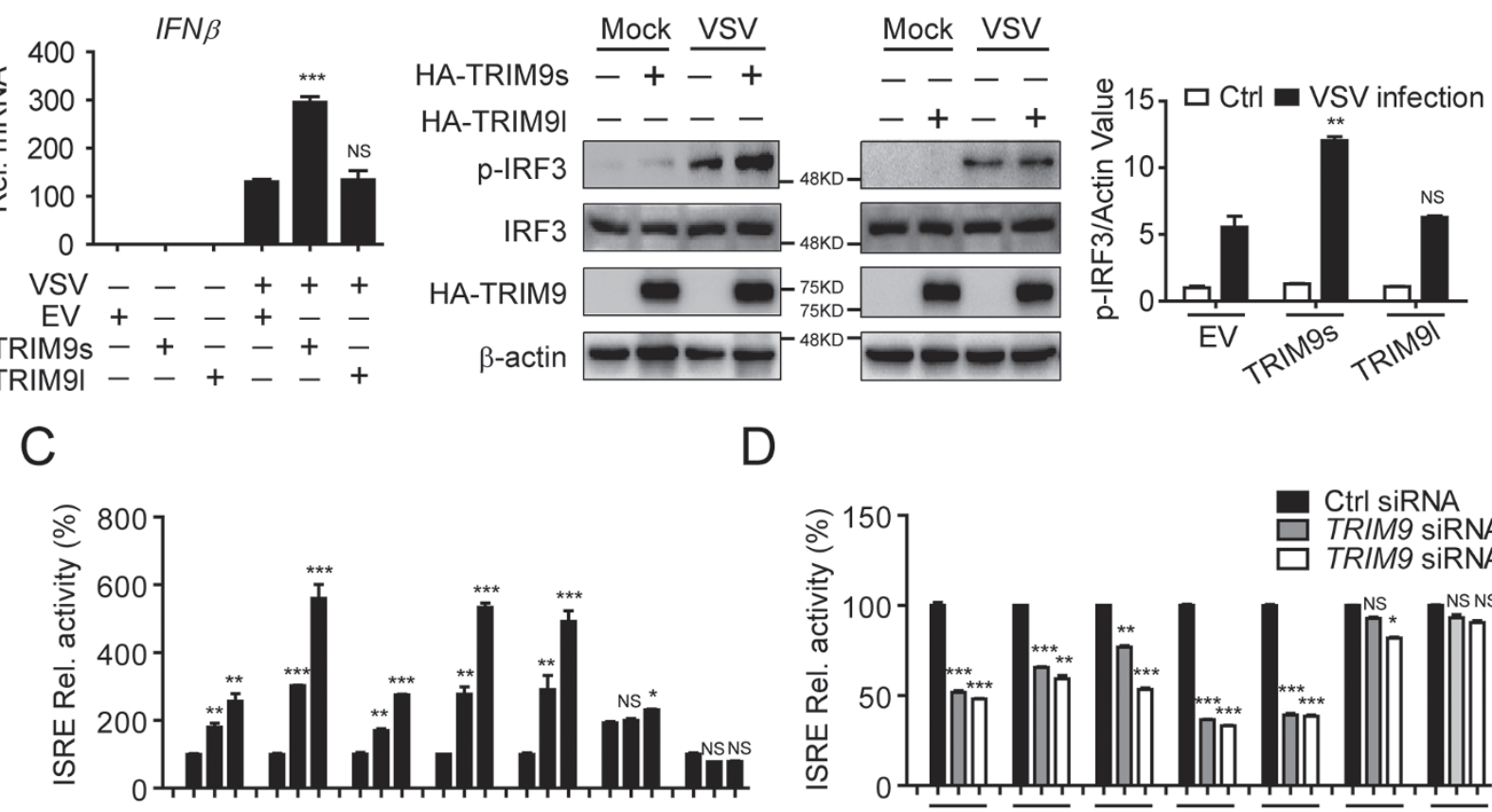

Myc-TRIM9s
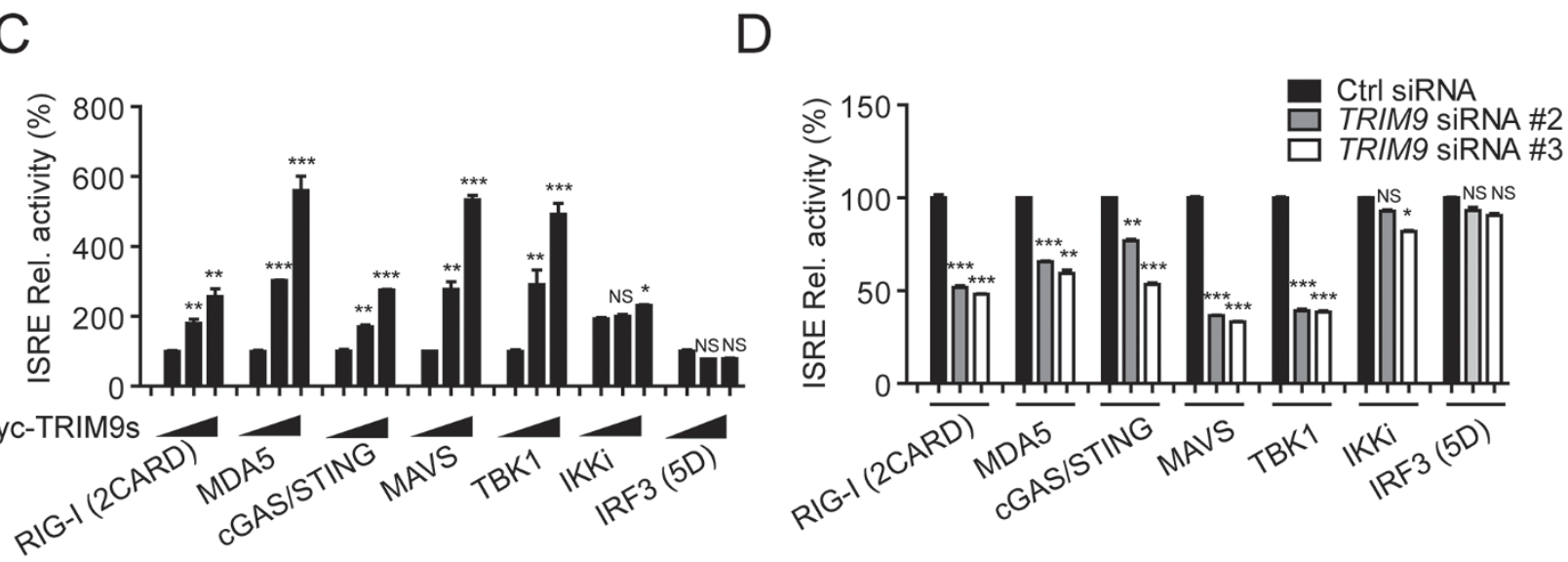

E

F
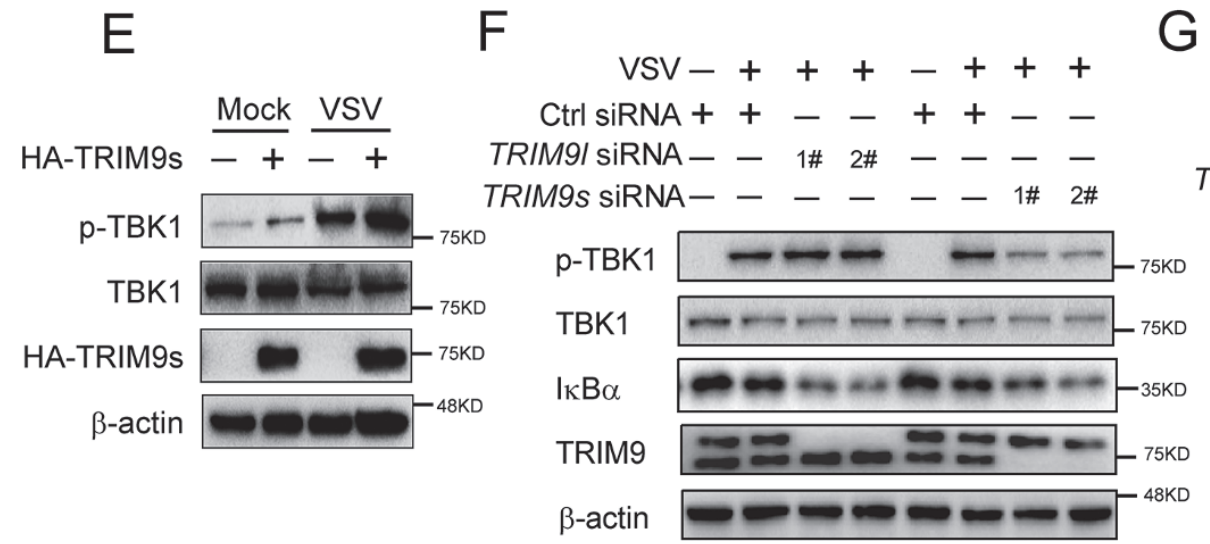

PBMC

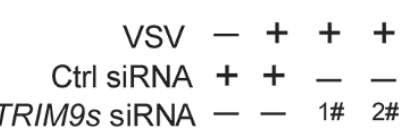

p-TBK1

TBK1

TRIM9s

$\beta$-actin

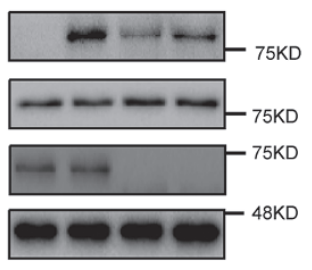

Figure 2 TRIM9s enhances IRF3 signaling through facilitating TBK1 phosphorylation. (A) Real-time PCR analysis of IFN $\beta$ mRNA in 293T cells that were transfected with TRIM9s, TRIM9I or empty vector (200 ng), followed by no infection (-) or infection (+) with VSV-eGFP (MOI, 0.05). The mRNA levels shown are relative to those of untreated cells. (B) IB analysis of total and phosphorylated ( $p-$ IRF3 in 293T cells treated as in A (left). The intensities of $p$-IRF3 and $\beta$-actin bands were quantified, and the relative $p$-IRF3/actin ratios were used to demonstrate the increase of p-IRF3 levels (right). (C) TRIM9s promotes RIG-I (2CARD)-, MDA5-, cGAS-, MAVS-, and TBK1-mediated ISRE reporter activation. 293T cells were transfected with the ISRE luciferase reporter, together with the indicated plasmids along with empty vector (no wedge) or increasing amounts (wedge) of expression vector for TRIM9s. (D) Knockdown of TRIM9 reduces RIG-I (2CARD)-, MDA5-, cGAS-, MAVS- and TBK1-mediated ISRE reporter activation. 293T cells were transfected with the ISRE luciferase reporter, together with the indicated plasmids, along with control (Ctrl) siRNA or TRIM9-specific siRNAs. (E-G) IB analysis of total and phosphorylated ( $p-$ ) TBK1 levels in 293T cells (E), A549 cells (F) and PBMCs (G) transfected with the indicated plasmids or siRNAs. ${ }^{*}<0.05$, ${ }^{* *} P<0.01$ and ${ }^{* * *} P<0.001$, versus the cells transfected with the empty vector $(\mathbf{A}, \mathbf{B})$ or overexpression of RIG-I, MDA5, MAVS, cGAS/STING, TBK1, IKKi, or IRF3 (5D) alone (C-D) (Student's $t$-test). Data are representative of three independent experiments (mean \pm SD in A-D). 
ISRE reporter activation by RIG-I (2CARD), MDA5, cGAS/STING, MAVS and TBK1 (Figure 2D). Consistent with these observations, we found that TRIM9s overexpression enhanced the phosphorylation of TBK1 (p-TBK1) after VSV infection (Figure 2E), whereas knockdown of endogenous TRIM9s, but not TRIM9l, severely impaired TBK1 phosphorylation in A549 cells or PBMCs (Figure 2F and 2G). Taken together, these results suggest that TRIM9s markedly enhances IRF3 signaling through facilitating TBK1 phosphorylation.

\section{TRIM9s interacts with TBK1 after viral infection}

Next, we sought to determine whether TRIM9s could directly interact with TBK1 or other signaling proteins within the type I IFN pathway. Co-immunoprecipitation experiments revealed that TRIM9s specifically interacted with TBK1, but not RIG-I, MDA5, MAVS, IKKi, IRF3, cGAS or STING (Figure 3A and Supplementary information, Figure S3C). To determine the physiological relevance of these findings, we treated 293T cells, A549 cells or PBMCs with VSV or HSV-1, and then collected the cell lysates at various time points. Immunoprecipitation and immunoblot analysis revealed that TRIM9s did not interact or only weakly interacted with TBK1 in unstimulated cells. However, TRIM9s strongly interacted with TBK1 after viral infection (Figure 3B3E). Since TRIM9s interacted with TBK1 only upon viral infection, we hypothesized that TRIM9s may only associate with the active form of TBK1. We generated TBK1 non-phosphorylatable mutants by substituting serine 172 with alanine (S172A) or lysine 38 with aspartic acid (K38D). Interestingly, we found that TRIM9s only interacted with WT TBK1, but not with TBK1 mutants (S172A or K38D) (Figure 3F). These results suggest that phosphorylation of TBK1 is required for its interaction with TRIM9s.

To identify the domain of TBK1 responsible for its interaction with TRIM9s, we generated three TBK1 deletion constructs (Figure 3G, top panel). We found that the kinase domain (KD) of TBK1 was critical for binding to TRIM9s (Figure 3G, bottom panel). Because the $\mathrm{KD}$ is necessary for TBK1 phosphorylation, our results further confirmed that TRIM9s interacts with phosphorylated TBK1. Next, we investigated which domain of TRIM9s is responsible for its interaction with TBK1 by generating four TRIM9s deletion mutants, TRIM9s- $\Delta$ RING (aa 164-550), TRIM9s-BB (aa 164-266), TRIM9s-RBB (aa 1-266) and TRIM9s-RBBC (aa 1-393) (Figure 3H, top). We found that the RING domain was essential for interacting with TBK1 (Figure 3H, bottom panel). These results indicate that TRIM9s enhances type I IFN activation via binding to $\mathrm{p}$-TBK1 through its RING domain.
TRIM9s bridges GSK3 $\beta$ and TBK1 to promote TBK1 oligomerization

After viral infection, GSK3 $\beta$ aids in the oligomerization of TBK1 to reach its maximum activity [18], and TRIM9s targets TBK1 and enhances its phosphorylation. Therefore, we reasoned that TRIM9s may serve as a platform for TBK1 association. First, we found that TRIM9s, but not TRIM91, markedly enhanced TBK1 oligomerization (Figure 4A and Supplementary information, Figure S4A). Next, we examined whether TRIM9s bridges GSK3 $\beta$ to TBK1. As expected, TRIM9s associated with GSK3 $\beta$ (Figure 4B), but TRIM91 did not (Supplementary information, Figure S4B). Moreover, we found that the specific C-terminal domain of TRIM9s was necessary for binding to GSK3 $\beta$ (Supplementary information, Figure S4C). Furthermore, co-immunoprecipitation and immunoblot analysis revealed that TRIM9s overexpression strengthened the association between GSK3 $\beta$ and TBK1 (Figure 4C). Knockdown of TRIM9s severely impaired the interaction between GSK3 $\beta$ and TBK1 (Figure 4D). During viral infection, the interaction between GSK3 $\beta$ and TBK 1 requires the presence of TRIM9s (Figure $4 \mathrm{E}$ ), and GSK3 $\beta$-induced TBK1 oligomerization was completely abolished in TRIM9s-KO cells (Figure 4F). Finally, we used $G S K 3 \beta$-specific siRNAs to knock down GSK $3 \beta$ (Figure $4 \mathrm{G}$ ) and found that under such conditions TRIM9s failed to enhance TBK1-induced ISRE reporter activation (Figure $4 \mathrm{H}$ ). Moreover, TRIM9s did not promote TBK1 oligomerization in GSK3 $\beta$-knockdown cells (Figure 4I). Collectively, these data suggest that TRIM9s bridges GSK $3 \beta$ and TBK1 to initiate TBK1 oligomerization and that GSK3 $\beta$ is essential for TRIM9s to promote TBK1 activation.

TRIM9 promotes TBK1-mediated signaling through its E3 ligase activity

It has been reported that the phosphorylation of TRIM9 at its RING domain (Ser76 and Ser80) is critical for its inhibitory effect on NF-kB signaling [28]. We examined whether TRIM9s-mediated type I IFN activation is also dependent on TRIM9s phosphorylation. We found that the TRIM9s-SA mutant (S76A/S80A) still interacted with TBK1 (Figure 5A). Consistently, the TRIM9s$\triangle$ RING mutant, but not TRIM9s-SA, lost the ability to enhance TBK1-mediated ISRE reporter activation, whereas neither TRIM9s- $\Delta$ RING nor TRIM9s-SA mutant inhibited TBK1-induced NF- $\mathrm{KB}$ activation (Figure $5 \mathrm{~B}$ and $5 \mathrm{C}$ ). These results suggest that the interaction between TRIM9s and TBK1 is independent of the phosphorylation at Ser76 and Ser80 of TRIM9s.

Since the RING domain of TRIM9s is critical for its function (Figure 5B and 5C), we examined whether the 


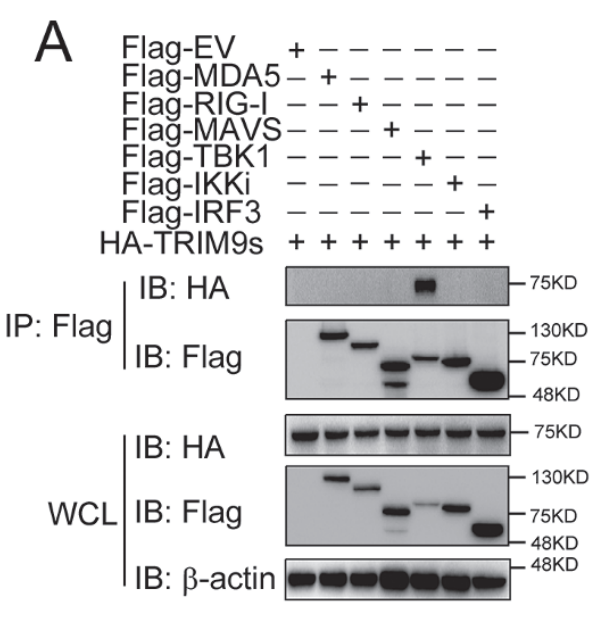

D
B 293T

$$
\begin{gathered}
\text { IP: } \\
\text { VSV(hr) }
\end{gathered} \frac{\operatorname{lgG}}{0} \frac{\text { TRIM9s }}{0 \quad 5 \quad 10}
$$

IB: TBK1

IB: TRIM9s

$$
\text { -75K }
$$

IB: TRIM9s

WCL

IB: TBK1

IB: $\beta$-actin

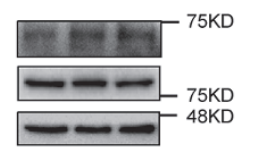

A549

$$
\text { IP: IgG TRIM9s }
$$

VSV $-\overline{-}+$

IB: TBK1

IB: TRIM9s ${ }^{75 \mathrm{kD}}$

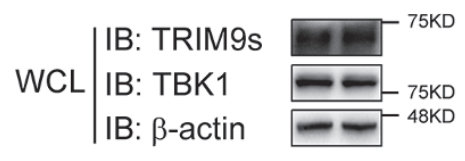

E Рвмс F

$$
\text { IP: IgG TRIM9s }
$$$$
\text { VSV }--+
$$

IB: TBK1 $\longrightarrow 75 \mathrm{KD}$

IB: TRIM9s

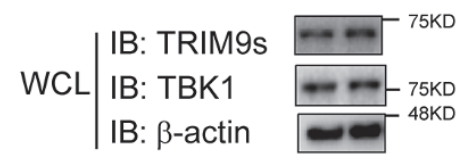

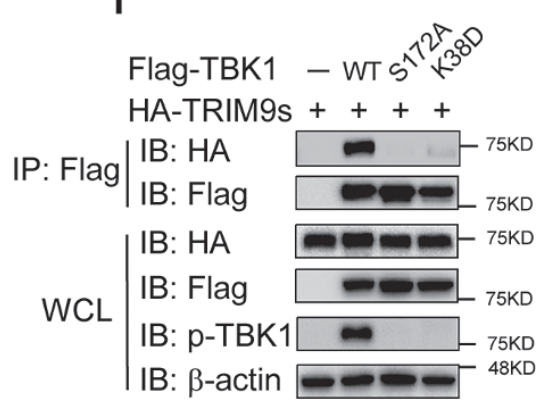

Flag-TBK1 - WT 1 $^{12}+\underbrace{8} 80$ HA-TRIM9s ++++ IB: Flag IB: TRIM9s WCL $\begin{aligned} & \text { IB: TRIM9s } \\ & \text { IB: TBK1 } \\ & \text { IB: } \beta \text {-actin }\end{aligned}$ $G$
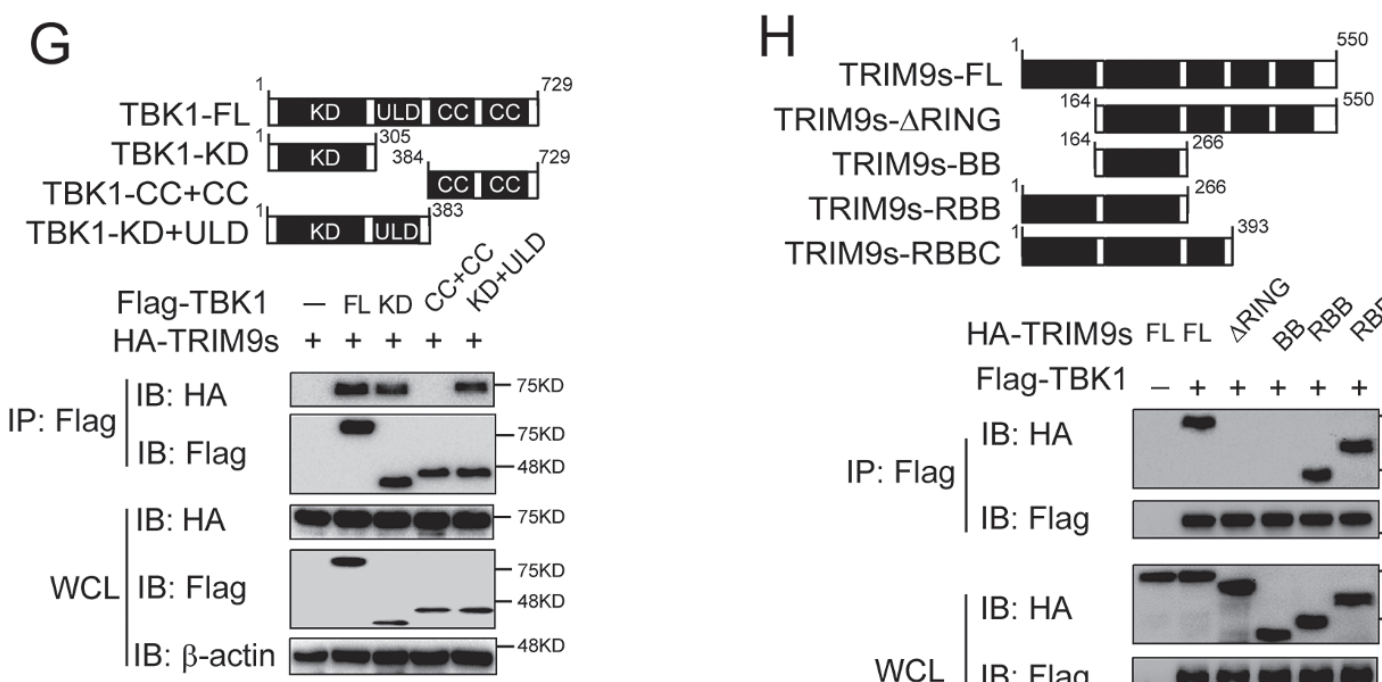

HA-TRIM9s FL FL

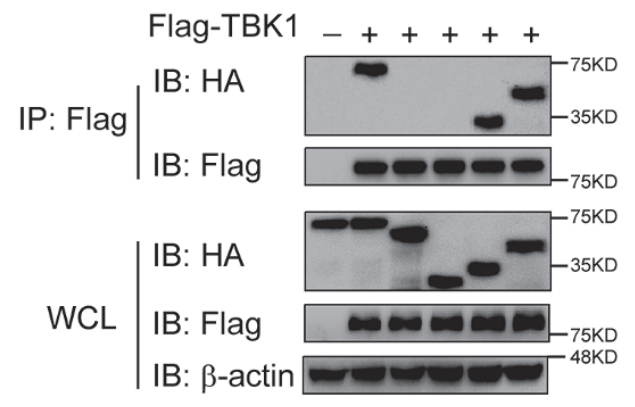

Figure 3 TRIM9s interacts with TBK1 upon viral infection. (A) Co-immunoprecipitation (Co-IP) and IB analysis of 293T cells transfected with various combinations (above lanes) of plasmids for Flag-MDA5, Flag-RIG-I, Flag-MAVS, Flag-TBK1, Flag-IKKi, Flag-IRF3, and HA-TRIM9s. (B-E) 293T cells (B), A549 cells (C, D) or PBMCs (E) were infected with VSV (B, C, E) or HSV-1 (D) and collected at the indicated time points. Cell extracts were harvested for IP with anti-TRIM9s, followed by IB analysis with anti-TBK1 antibody. (F) 293T cells were transfected with HA-TRIM9s and Flag-TBK1 or various mutants. Whole cell extracts were immunoprecipitated with anti-Flag beads, followed by IB analysis with anti-HA antibody. (G) Co-IP and IB analysis (bottom) of 293T cells transfected with TBK1 deletion mutants (top) along with HA-TRIM9s. WT, wild-type; KD, kinase domain; ULD, ubiquitin-like domain; CC, coiled-coil domain. (H) 293T cells were transfected with Flag-TBK1 and the deletion mutants of TRIM9s (top). Whole-cell extracts were immunoprecipitated with anti-Flag beads, followed by IB analysis with anti-HA antibody. Data are representative of three independent experiments with similar results. 

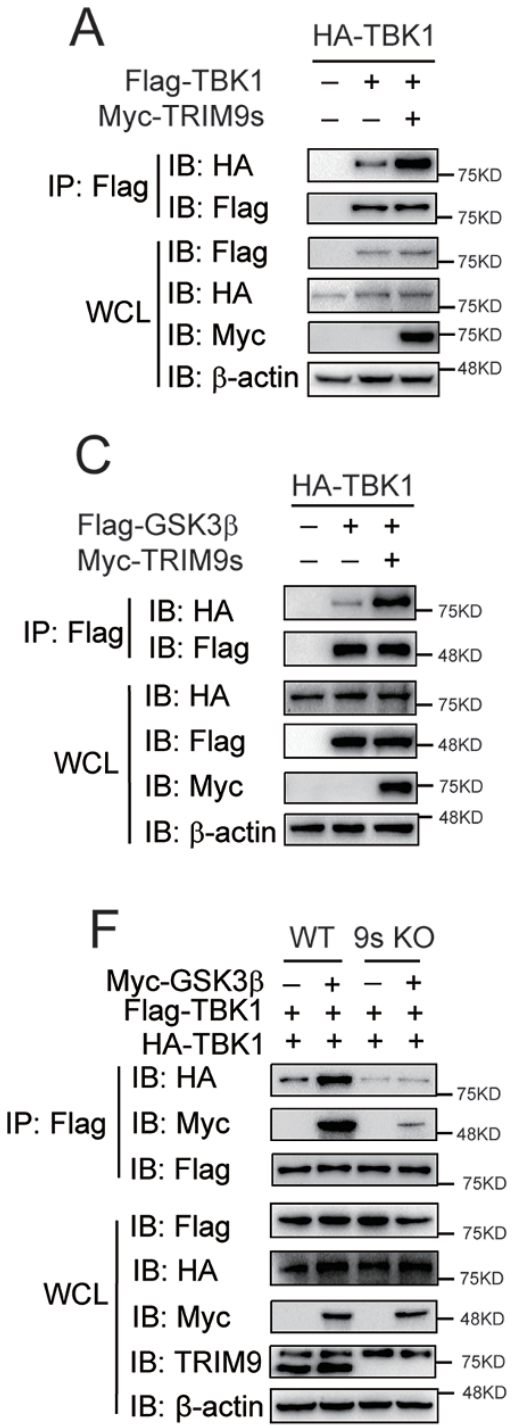

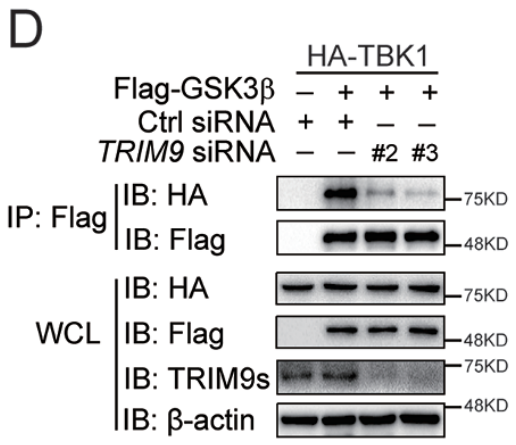

G

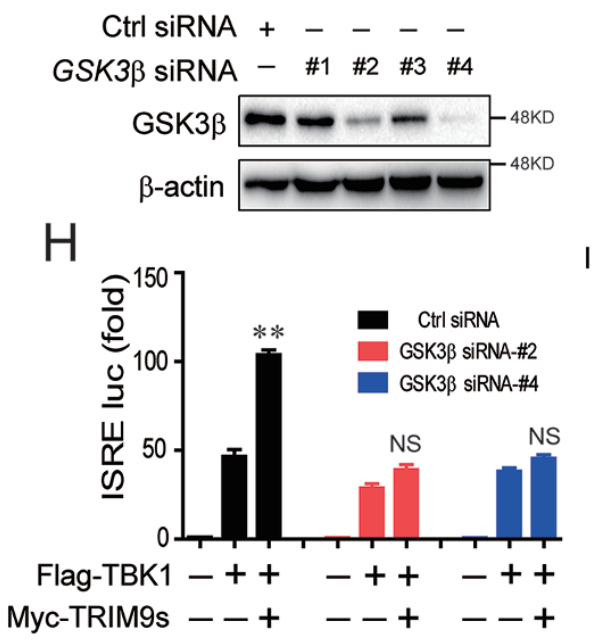

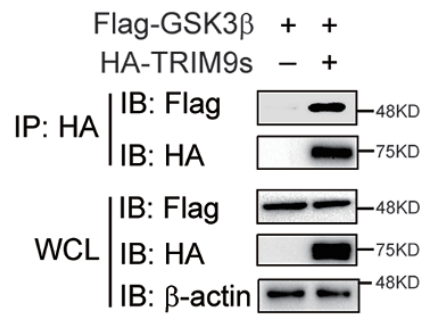

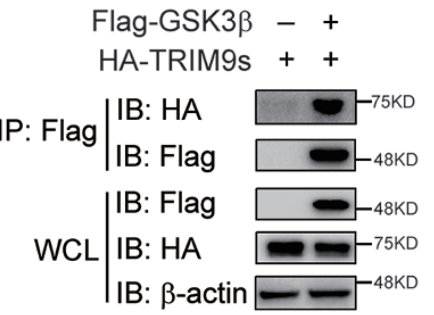

$E$

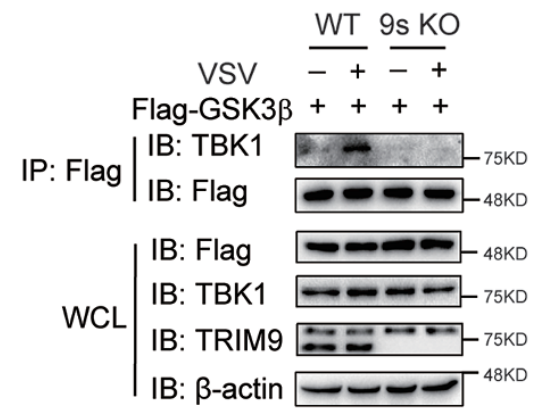

|

\begin{tabular}{|c|c|c|c|}
\hline Flag-TBK1 & + & + & \\
\hline HA-TBK1 & + & + & + \\
\hline Myc-TRIM9s & - & + & + \\
\hline Ctrl siRNA & + & + & - \\
\hline GSK3 $\beta$ siRNA & - & - & \# \\
\hline
\end{tabular}

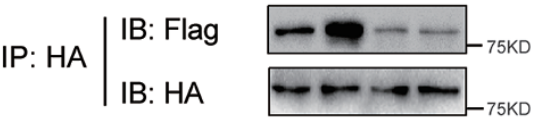

$\left.W_{\text {WCL }}\right|_{\text {IB: } \mathrm{HA}}$

Figure 4 TRIM9s bridges GSK3 3 to TBK1. (A) 293T cells were transfected with HA- and Flag-TBK1, with or without MycTRIM9s. Cell extracts were collected for IP with anti-Flag beads, followed by IB analysis with anti-HA antibody. (B) Co-IP and IB analysis of 293T cells transfected with Flag-GSK3 $\beta$ and HA-TRIM9s. (C) 293T cells were transfected with HA-TBK1 and Flag-GSK3 $\beta$, with or without Myc-TRIM9s. Cell extracts were collected for IP with anti-Flag beads, followed by IB analysis with anti-HA antibody. (D) 293T cells were transfected with HA-TBK1 and Flag-GSK3 $\beta$, along with control (Ctrl) siRNA or TRIM9-specific siRNAs. Cell extracts were collected for IP with anti-Flag beads, followed by IB analysis with anti-HA antibody. (E) WT and 9s KO 293T cells were transfected with Flag-GSK3 $\beta$, followed by VSV infection. Cell extracts were collected for IP with anti-Flag beads, followed by IB analysis with anti-TBK1 antibody. (F) WT and TRIM9s KO 293T cells were transfected with Flag- and HA-TBK1, along with or without Myc-GSK3 3 . Cell extracts were collected for IP with anti-Flag beads, followed by IB analysis with anti-HA and anti-Myc antibodies. (G) Knockdown efficiency of GSK3 $\beta$ in 293T cells with GSK3 $\beta$-specific siRNAs or control siRNA. (H) 293T cells were transfected with control or GSK3 $\beta$ siRNAs for $24 \mathrm{~h}$, followed by the transfection with an ISRE luciferase reporter, together with Flag-TBK1 plasmids along with empty vector or expression vector for MycTRIM9s. (I) 293T cells were transfected with control or GSK3 $\beta$ siRNAs for $24 \mathrm{~h}$. The cells were then transfected with the indicated plasmids. Cell extracts were collected for IP with anti-HA beads, followed by IB analysis with anti-Flag antibody. $* P<$ $0.05,{ }^{* *} P<0.01$ and ${ }^{* * *} P<0.001$, versus the cells transfected with Flag-TBK1 and control siRNA (Student's $t$-test). Data are representative of three independent experiments (mean $\pm \mathrm{SD}$ in $\mathbf{H}$ ). 
A

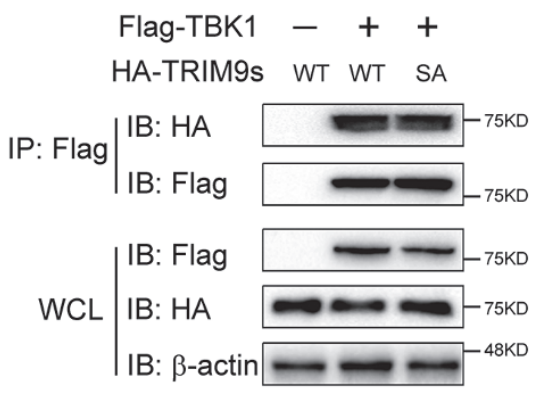

D

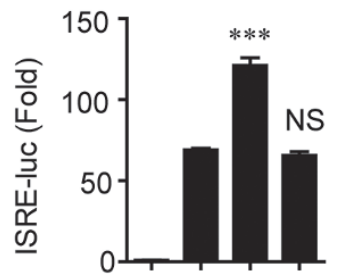

Flag-TBK1 -+++ Myc-TRIM9s --+Myc-TRIM9s-CA --++
B

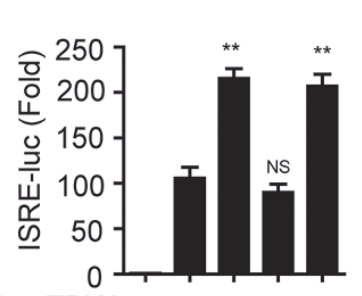

Flag-TBK $1-++++$ HA-TRIM9s --+HA-TRIM9s- $\triangle$ RING ---+ HA-TRIM9S-SA ---+

E

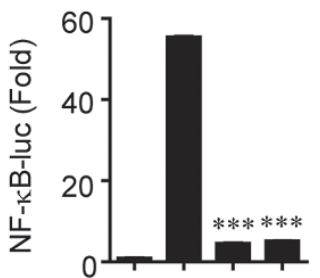

Flag-TBK1 -+++ Myc-TRIM9s --+Myc-TRIM9s-CA ---+
C

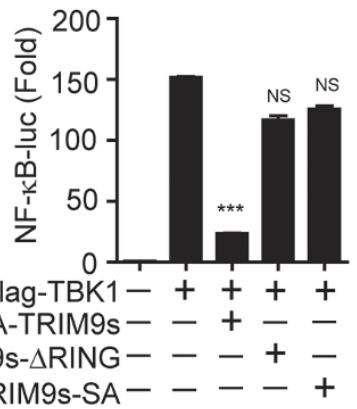

HA-TRIM9s- $\triangle$ RING --+-
HA-TRIM9s-SA ---+

$\mathrm{F}$

$$
\begin{aligned}
& \text { Flag-TBK1 }+++ \\
& \text { HA-TRIM9s }- \text { WT CA }
\end{aligned}
$$

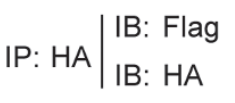

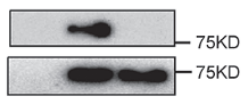

WCL $\begin{aligned} & \text { IB: } \mathrm{HA} \\ & \text { IB: Flag } \longrightarrow-75 \mathrm{KD} \\ & \text { IB: } \beta \text {-actin }\end{aligned}$

Figure 5 TRIM9 promotes TBK1-mediated signaling through its E3 ligase activity. (A) 293T cells were transfected with FlagTBK1 and WT HA-TRIM9s or its mutant HA-TRIM9s (SA). Whole-cell extracts were immunoprecipitated with anti-Flag beads, followed by IB analysis with anti-HA. (B, C) 293T cells were transfected with the ISRE (B) or NF- $\mathrm{B}$ luciferase reporter (C), together with TBK1 along with empty vector, WT TRIM9s, TRIM9s ( $R$ RING) or TRIM9s (SA). (D, E) 293T cells were transfected with the ISRE (D) or NF-KB (E) luciferase reporter, together with TBK1 along with empty vector, WT TRIM9s or TRIM9s (CA). (F) 293T cells were transfected with Flag-TBK1 and HA-TRIM9s or TRIM9s (CA) for $24 \mathrm{~h}$. Cell lysates were immunoprecipitated by anti-HA beads, followed by IB analysis with anti-Flag. $* P<0.05, * * P<0.01$ and $* * * P<0.001$, versus the cells transfected with the TBK1 alone (Student's $t$-test). Data are representative of three independent experiments (mean \pm SD in B-E).

E3 ligase activity of TRIM9s is essential for its function. An enzymatically inactive mutant TRIM9s-CA (C30A/ C33A) could not facilitate ISRE reporter activation, but still inhibited NF- $\kappa$ B activation (Figure $5 \mathrm{D}$ and $5 \mathrm{E}$ ), suggesting that TRIM9s may affect the NF- $\mathrm{kB}$ and IRF3 pathways through distinct mechanisms. In addition, immunoprecipitation and immunoblot analysis showed that TRIM9s-CA failed to bind to TBK1 (Figure 5F), suggesting that the E3 ligase activity of TRIM9s is required for its interaction with TBK1.

Auto-regulated K63-linked ubiquitination of TRIM9s is required for its interaction with TBK1

Next, we tested whether TRIM9s affects the ubiquitination of TBK1 and other signaling proteins. Although it has been reported that the K63-linked ubiquitination of TBK1 is important for the activation of type I IFN signaling $[13,14]$, we did not observe any changes in WT, K63-, or K48-linked ubiquitination of TBK1 or GSK3 $\beta$ in WT or TRIM9s KO cells (Supplementary information,
Figure S5A and S5B), which suggests that neither TBK1 nor GSK3 $\beta$ is a direct substrate of TRIM9s.

Recently, TRIM14 has been reported to positively regulate RIG-I signaling by recruiting NEMO through its K63-linked poly-ubiquitin chains and consequently linking NEMO to MAVS [30]. We observed enhanced oligomerization of TRIM9s after viral infection (Figure 6A), indicating that TRIM9s may act as its own target for ubiquitination. Consistent with our hypothesis, we found that TRIM9s undergoes robust polyubiquitination upon VSV or HSV-1 infection (Figure 6B and Supplementary information, Figure S5C). Further experiments showed that VSV infection substantially increased the polyubiquitination of TRIM9s with K63 linkage, but not with K48 linkage, (Supplementary information, Figure S6A). Previous studies showed that TRIM9 can be auto-ubiquitinated [26]. Our results revealed that the polyubiquitination of TRIM9s-CA is much weaker compared with WT TRIM9s (Figure 6C), whereas the ubiquitination of TRIM9s-CA could be restored in the presence of WT TRIM9s (Fig- 


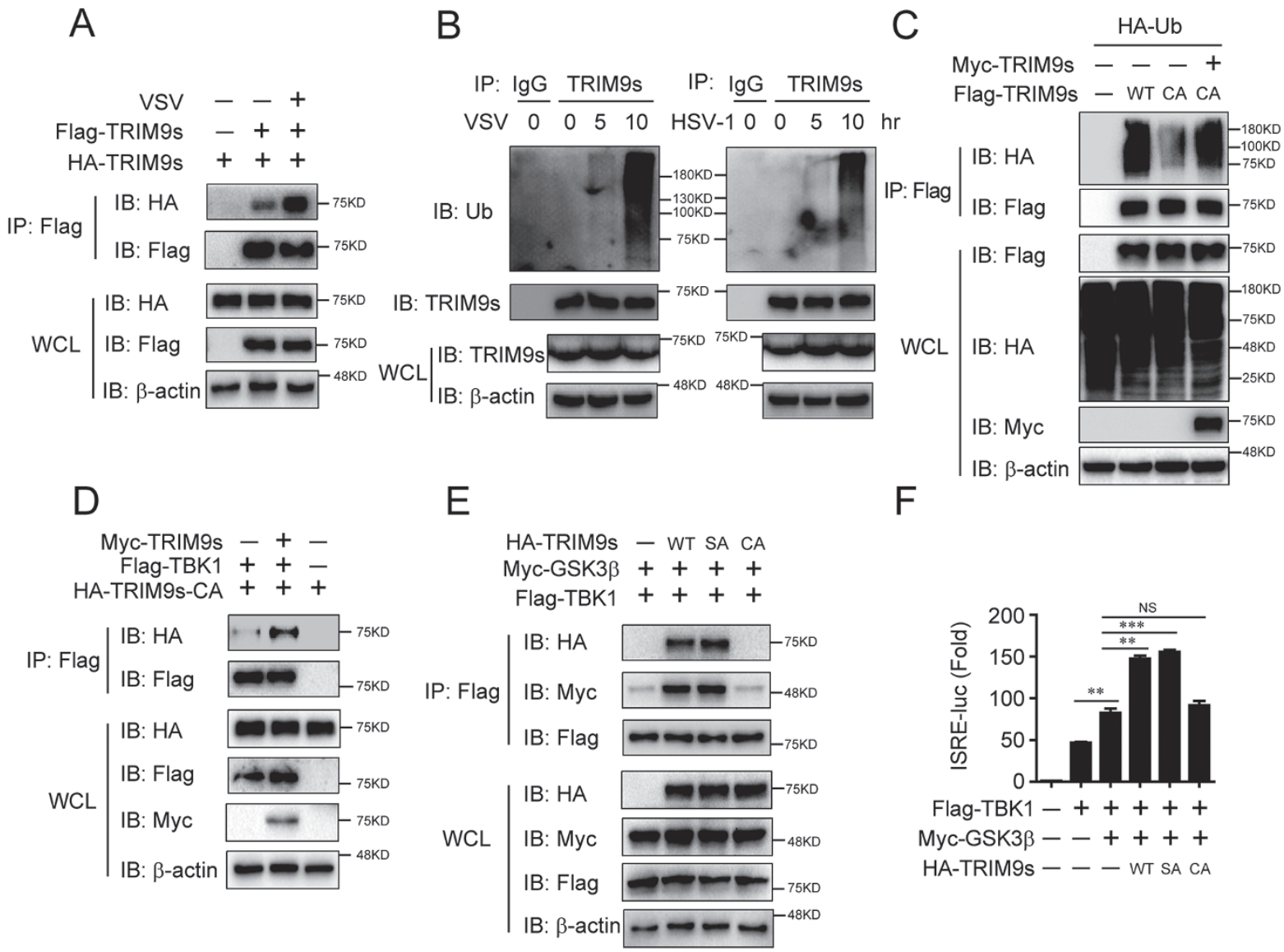

Figure 6 Auto-regulated K63-linked ubiquitination of TRIM9s is required for its interaction with TBK1. (A) 293T cells were transfected with HA- and Flag-TRIM9s, followed by VSV infection and IP with anti-Flag beads and IB analysis with HA. (B) A549 cells were infected with VSV or HSV-1 for the indicated time points. Cell lysates were immunoprecipitated using anti-TRIM9s antibody or IgG, followed by IB analysis with the indicated antibodies. (C) 293T cells were transfected with HA-Ub, Flag-TRIM9s, Flag-TRIM9s (CA) alone, or Flag-TRIM9s (CA) together with Myc-TRIM9s. Cell lysates were immunoprecipitated by anti-Flag beads, followed by IB analysis with anti-HA. (D) 293T cells were transfected with Flag-TBK1, HA-TRIM9s (CA), with or without Myc-TRIM9s. Cell lysates were immunoprecipitated by anti-Flag beads, followed by IB analysis with anti-HA. (E) 293T cells were transfected with Flag-TBK1, Myc-GSK3 $\beta$ and HA-TRIM9s or its mutants. Cell lysates were immunoprecipitated by anti-Flag beads, followed by IB analysis with anti-HA and anti-Myc. (F) 293T cells were transfected with the ISRE luciferase reporter, together with TBK1 and GSK3 $\beta$, along with empty vector, WT TRIM9s, TRIM9s (SA), or TRIM9s (CA). **P < 0.01 and $* * * P<0.001$, versus the cells transfected with TBK1 alone or transfected with TBK1 and GSK3 $\beta$ (Student's $t$-test). Data are representative of three independent experiments (mean \pm SD in F).

ure $6 \mathrm{C})$. We also observed that the oligomerization of TRIM9s-CA was weak, compared with the interaction between TRIM9s-CA and TRIM9s-WT or auto-oligomerization of TRIM9s-WT (Supplementary information, Figure S6B), indicating that TRIM9s can initiate oligomerization in the absence of ubiquitination while ubiquitination can further enhance TRIM9s oligomerization. These results suggest that TRIM9s undergoes oligomerization and K63linked auto-ubiquitination after viral infection.

Next we examined whether the ubiquitination of TRIM9s is required for its binding ability to TBK1.
Co-immunoprecipitation experiments showed that TRIM9s-CA alone did not bind to TBK1, but the interaction could be restored in the presence of WT TRIM9s (Figure 6D). This result suggests that auto-ubiquitination of TRIM9s is essential for the recruitment of TBK1. We further found that TRIM9s-CA failed to bridge GSK3 $\beta$ to TBK1 (Figure 6E) or to facilitate GSK3 $\beta$-mediated TBK1 activation (Figure 6F). Collectively, these results suggest that viral infection triggers the K63-linked auto-polyubiquitination of TRIM9s, providing the platform to bridge GSK3 $\beta$ to TBK1. 
TRIM9s balances IRF3 and NF- $\kappa B$ signaling upon viral infection

Viral infection often induces the antiviral response and inflammation through the IRF3 and NF- $\mathrm{BB}$ pathways, respectively. Recently, TRIM91 has been reported to be a negative regulator of $\mathrm{NF}-\kappa \mathrm{B}$-dependent pro-inflammatory signaling through inhibition of $\beta-\mathrm{TrCP}$ activity [28]. To investigate how TRIM9 regulates IRF3 and NF- $\mathrm{B}$ signaling in antiviral immunity, we examined the functions of TRIM9s and TRIM91 in RIG-I- and cGAS-triggered NF- $\kappa B$ or IRF3 activation. As expected, both TRIM9s and TRIM91 inhibited RIG-I- and cGAS-induced NF$\kappa \mathrm{B}$ activation, but only TRIM9s enhanced RIG-I- and cGAS-induced ISRE reporter activity (Figure 7A). Upon viral infection, knockout of TRIM9s promoted virus-induced I $\mathrm{B} \alpha \alpha$ degradation, and impaired TBK1 and IRF3 phosphorylation simultaneously (Figure 7B).

Next, we investigated whether the regulatory effect of TRIM9s on IRF3 and NF- $\kappa$ B pathways leads to enhanced production of type I IFNs and suppressed production of pro-inflammatory cytokines. We infected doxycycline-inducible A549-TRIM9s cells with VSV or HSV-1 and found that doxycycline-induced overexpression of TRIM9s significantly upregulated $I F N \alpha-8, I F N \beta$ and ISG54 expression, but downregulated the expression of pro-inflammatory cytokines, such as $T N F \alpha$ and $I L-6$ (Figure 7C). Moreover, knockdown of TRIM9s impaired the expression of $I F N \alpha-8, I F N \beta$ and $I S G 54$, but enhanced the expression of $T N F \alpha$ and $I L-6$ (Figure 7D). Similar findings were obtained using TRIM9s KO cells (Figure 7E). Taken together, these results suggest that TRIM9s acts as a balancer to regulate NF- $\mathrm{kB}$ and IRF3 signaling, skewing innate immune responses towards type I IFN signaling.

\section{Discussion}

Accumulating evidence suggests that members of the TRIM family play versatile roles in inflammation and antiviral immunity $[19,25]$. It has been reported that many TRIMs affect the ubiquitination states of several key signaling molecules in NF- $\mathrm{kB}$ and type I IFN signaling via their E3 ligase activities [25, 36]. For example, TRIM $30 \alpha$ interacts with and degrades TAB2 and TAB3 through the ubiquitin-lysosome pathway, which is dependent on the E3 ligase activity of TRIM30 $\alpha$ [37]. Both TRIM32 and TRIM56 have been reported to positively regulate STING-mediated signaling by promoting K63linked ubiquitination of STING, albeit at different lysine residues $[22,23]$. TRIM38 negatively regulates TLR3/4and RIG-I-mediated IFN $\beta$ production by promoting K48-linked polyubiquitination and proteasomal degra- dation of NAP1 [38], whereas TRIM23-mediated K27linked ubiquitination of NEMO is essential for TLR3and RIG-I/MDA5-mediated antiviral and inflammatory responses [39]. In this study, overexpression, RNAi and KO systems consistently demonstrated that TRIM9s acts as a positive regulator in antiviral immunity, promoting IRF3 activation and type I IFN production upon viral infection. Moreover, biochemical analyses showed stimuli- and E3 ubiquitin ligase activity-dependent interaction of TRIM9s with TBK1 and activation of TBK1 by TRIM9s-mediated bridging of GSK3 $\beta$ to TBK1.

A recent study identified TRIM14 as an adaptor in MAVS-induced IRF3- and NF- $\kappa$ B-regulated pathways through its K63-linked polyubiquitination [30]. Upon viral infection, TRIM14 undergoes K63 polyubiquitination and recruits NEMO to MAVS. In addition, TRIM proteins serve as platforms for assembly of core autophagic factors in cellular autophagy [40], suggesting that TRIM proteins could serve as platforms for the assembly of essential signaling molecules in innate immunity. Similar to TRIM14, TRIM9s bridges GSK3 $\beta$ to TBK1 in an auto-ubiquitination-dependent manner. A previous report showed that TRIM9 could be auto-ubiquitinated using UBCH5b as the E2 enzyme [26]. In this study, we found that TRIM9s undergoes auto-ubiquitination and oligomerization after viral infection. Furthermore, the auto-ubiquitination of TRIM9s is critical for its function in activating IRF3, and its inactive mutant (C30A/C33A) fails to bind TBK1 to facilitate the assembly of TBK1 and GSK3ß.

It is well-documented that viral infection often triggers both IRF3 and NF- $\kappa$ B signaling cascades. Many regulators of type I IFN signaling, such as NLRC5 and NLRX1, function similarly in both IRF3 and NF$\kappa \mathrm{B}$ signaling pathways [41-43]. Interestingly, TRIM9s plays different roles in the regulation of IRF3 and NF- $\mathrm{kB}$ pathways. Unlike IFN $\alpha$, the production of IFN $\beta$ requires coordinated actions of the transcription factors, IRF3 and NF- $\kappa B$. Whether TRIM9s promotes or inhibits IFN $\beta$ production may depend on its optimal concentration. In this study, we found that TRIM9s positively regulates IFN $\beta$ production in $293 \mathrm{~T}$ and A549 cells. Whether TRIM9s functions similarly in other types of cells needs further investigation. Similarly, another E3 ligase, Nrdp1, inhibits NF- $\kappa B$, but enhances IRF3 signaling through targeting MyD88 and TBK1, respectively [14]. These types of regulators may act as balancers of innate immunity, switching the immune responses from "inflammation signaling" to "type I IFN signaling". Based on these experimental data, we propose a working model to illustrate how TRIM9s could balance virus-triggered NF- $\mathrm{KB}$ and IRF3 signaling pathways (Figure 8). After viral in- 


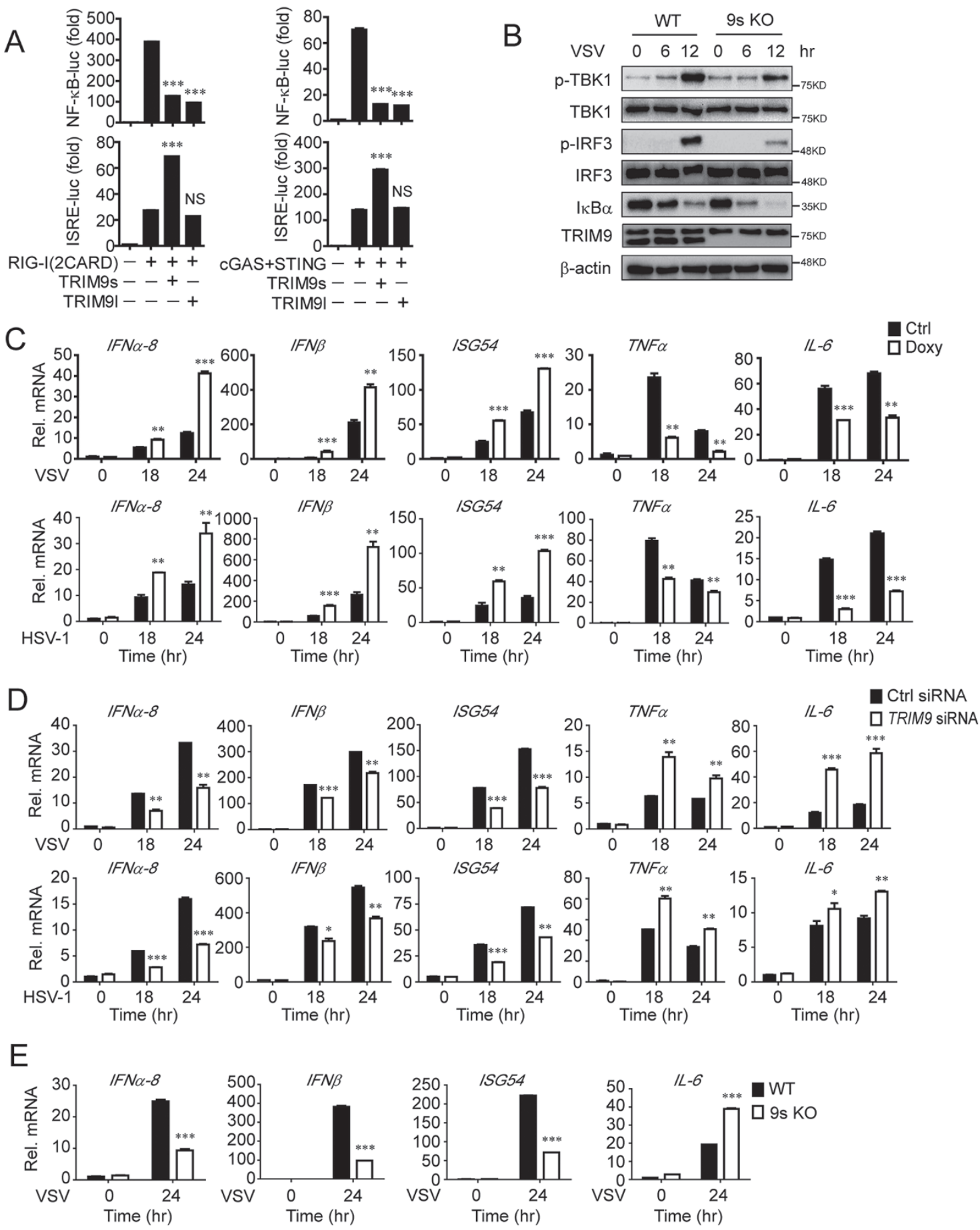

Figure 7 TRIM9s balances IRF3 and NF-kB signaling upon viral infection. (A) Luciferase activity in 293T cells transfected with plasmids encoding the luciferase reporter for NF-kB or ISRE, together with empty vector, TRIM9s or TRIM9I, along with RIG-I (2CARD; left) or cGAS/STING (right). Data are presented relative to Renilla luciferase activity. (B) WT or 9s KO A549 cells were infected with VSV-eGFP. The cell extracts were harvested at different time points for IB analysis. (C) A549-TRIM9s cells with Doxy-inducible TRIM9s expression were left untreated or treated with Doxy $(100 \mu \mathrm{g} / \mathrm{ml})$ for $24 \mathrm{~h}$, and then infected with VSV (MOI $=0.01)$ or HSV$1(\mathrm{MOI}=0.1)$ for 18 and $24 \mathrm{~h}$. The total mRNA was harvested, and the indicated mRNAs were analyzed by q-PCR. (D) Real-time PCR analysis of the indicated genes in A549 cells transfected with control siRNA or TRIM9 siRNA, followed by infection of VSV or HSV-1. (E) Real-time PCR analysis of the indicated genes in WT or 9s KO cells, followed by infection of VSV or HSV-1. $* P<0.05$, $* * P<0.01$ and $* * * P<0.001$, versus the cells transfected with RIG-I (CARD) or cGAS/STING (A) or the control cells with the same treatment (C-E) (Student's $t$-test). Data are representative of three independent experiments (mean $\pm S D$ in $\mathbf{A}$ and $\mathbf{C}$-E). 


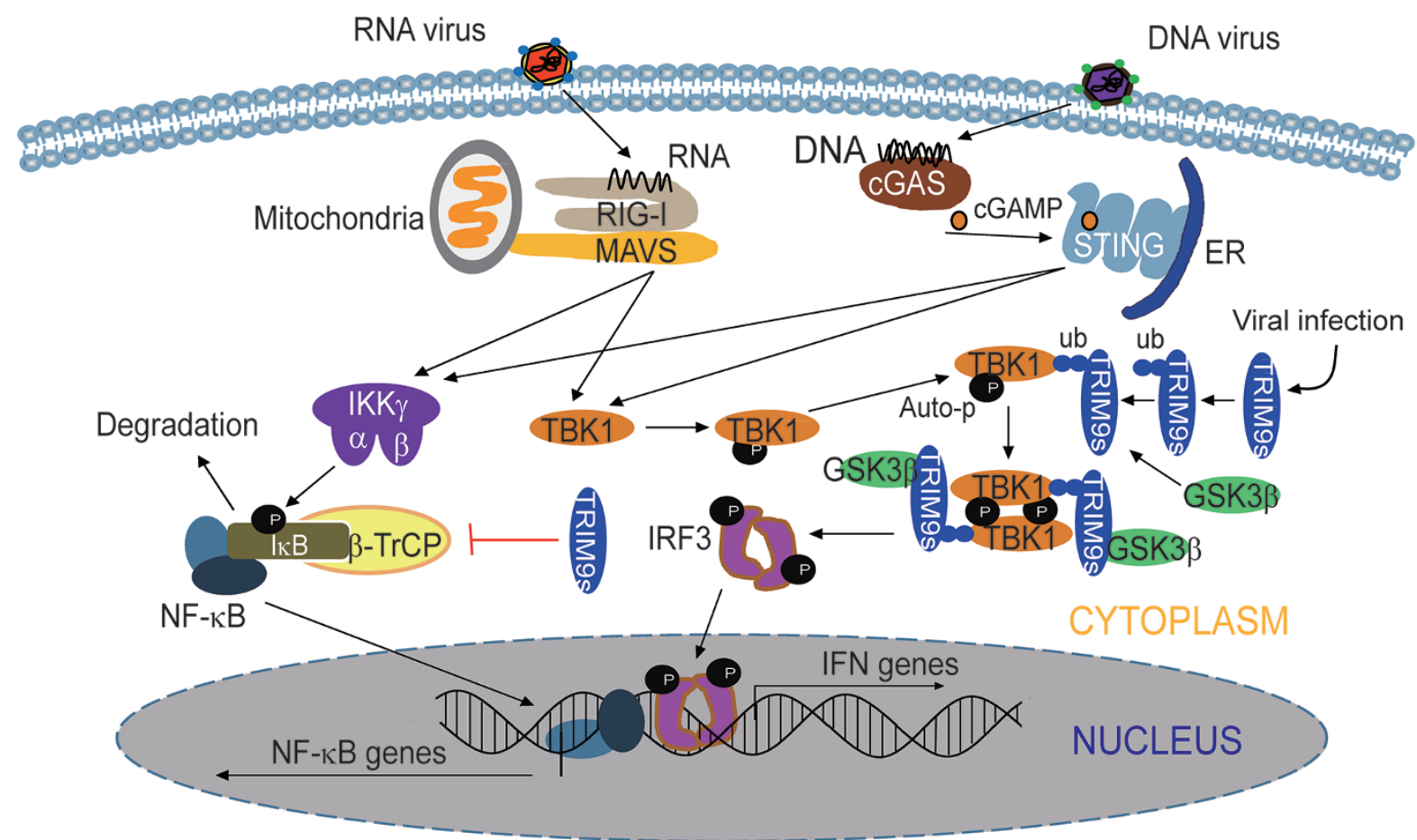

Figure 8 Working model of the regulation of the type I interferon and NF- $\kappa B$ signaling pathway by TRIM9s. Upon virus infection, TBK1 is activated by adaptor molecules, such as MAVS and STING, phosphorylates IRF3 and provokes strong IFN production. On the other hand, these adaptor molecules activate IKK complex to promote NF- $\mathrm{KB}$ signaling. In the process, TRIM9s undergoes oligomerization and K63-linked auto-ubiquitination. Ubiquitinated TRIM9s serves as a platform to bridge GSK3 $\beta$ to TBK1, thus enhancing the oligomerization and auto-phosphorylation of TBK1. On the other hand, TRIM9s inhibits $\mathrm{I}_{\kappa} \mathrm{B}$ degradation by suppressing $\beta$-TrCP activity, which leads to compromised NF- $\mathrm{KB}$ signaling and decreased production of pro-inflammatory cytokines.

fection, TRIM9s molecules associate with each other and undergo K63-linked auto-ubiquitination. Ubiquitinated TRIM9s provides a platform to bridge GSK3 $\beta$ to TBK1, thus initiating the association and subsequent activation of TBK1. On the other hand, TRIM9s strongly prevents $\mathrm{I} \kappa \mathrm{B}$ degradation by suppressing $\beta$-TrCP activity, which leads to decreased production of pro-inflammatory cytokines.

Our data suggest that TRIM9s and TRIM91 have distinct functions in the regulation of the IRF3 pathway. In this study, we observed the expression of two TRIM9 isoforms in the tissues of lung, liver and spleen or PBMC, U251, 293T, A549, THP-1 cells. TRIM9s has strong anti-viral ability, which was confirmed by overexpression, knockdown and KO experiments. However, TRIM91 can inhibit viral infection only when it was highly overexpressed, suggesting that TRIM9 long isoform may act in IFN pathways in certain conditions as previously reported by Versteeg et al. [25]. We speculate that TRIM9l's function is relatively limited because it did not recruit GSK3 $\beta$ to promote TBK1's oligomerization. We also noted that there were three reported isoforms of TRIM9 in the Uniprot database, but we failed to detect the third one in the tissues or cells that we examined. It is possible that the third isoform of TRIM9 might be expressed in other tissues and cells, or its abundance is too low to be detected. Further studies will be needed to illuminate the function of that isoform. Similar to TRIM9, several TRIM19/PML isoforms are generated from a single PML gene. They are homologous in their $\mathrm{N}$-terminal region but differ in their C-terminal sequences, and only PMLIII and PMLIV, but not the other isoforms, confer resistance to viral infection. However, PMLIV is the only PML isoform that is able to increase IFN $\beta$ production through IRF3 activation [44]. Since $\sim 90 \%$ of TRIM proteins have more than one splicing variants [25], re-evaluating their functions may be a worthwhile endeavor.

In summary, our findings provide insights into the molecular mechanisms by which TRIM9s positively regulates IRF3 signaling. TRIM9s thus plays a critical role in maintaining the balance between NF- $\kappa \mathrm{B}$-mediated pro-inflammatory signaling and IRF3-mediated type I 
IFN signaling. Type I IFNs, especially IFN $\alpha$, are critical cytokines involved in cancer treatment, whereas chronic inflammation is considered a risk factor for many types of cancer. Given its dual roles in regulating the production of type I IFNs and inflammatory cytokines, TRIM9s may serve as a useful therapeutic target for future cancer treatment.

\section{Materials and Methods}

\section{Constructs}

$\mathrm{NF}-\kappa \mathrm{B}$, ISRE promoter luciferase reporter plasmids, mammalian expression plasmids for HA- or Flag-tagged RIG-I (2CARD), MDA5, STING, RIG-I, STING, MAVS, TBK1, IKKi, IRF3 and TBK1 have been previously described [41]. TRIMs expression plasmids were constructed by GATEWAY system from the cDNA library (Invitrogen). Mammalian expression plasmids for human HA-tagged GSK3 $\beta$ and Flag-IRF3 (5D) were provided by Xiaofeng Qin (Sun Yat-sen Universty). cGAS, Flag (or Myc)-GSK3 $\beta$, TRIM9s-SA, TRIM9s-CA, TBK1 and TRIM9s domain deletions were constructed using standard molecular biology techniques.

\section{Antibodies}

The following antibodies were used in this study: anti-IRF3 (sc-9082), donkey anti-goat IgG-HRP (sc-2020), goat anti-rabbit IgG-HRP (sc-2004), goat anti-mouse IgG-HRP (sc-2005) (Santa Cruz Biotechnology); horseradish peroxidase (HRP)-anti-Flag (M2) (A8592) and anti- $\beta$-actin (A1978) (Sigma); HRP-anti-hemagglutinin (clone 3F10), anti-Myc-HRP (11814150001), unlabeled anti-Myc (11667203001) (Roche Applied Science); anti-pIRF3 (\#4947S), anti-TBK1 (\#3013), anti-TBK1 phosphorylated at Ser172 (\#5483) (Cell Signaling Technology); anti-TRIM9 (detects both TRIM9s and TRIM91; 10786-1-AP; Proteintech); anti-TRIM9s (detects only TRIM9s; TA800044; OriGene).

\section{Virus infection}

HSV-1 (KOS strain) was kindly provided by Dr Guoying Zhou (Guangzhou Medical University). VSV-eGFP was kindly provided by Dr Xiaofeng Qin (Sun Yat-sen University). Cells were infected at various MOI, as previously described [15].

\section{Luciferase and reporter assays}

HEK293T $\left(2 \times 10^{5}\right)$ cells were plated in 24-well plates and transfected, using Lipofectamine 2000 (Invitrogen), with plasmids encoding an ISRE or NF- $\mathrm{BB}$ luciferase reporter (firefly luciferase; $100 \mathrm{ng}$ ) and pRL-TK (renilla luciferase plasmid; $10 \mathrm{ng}$ ) together with 100 ng plasmid encoding Flag-RIG-I (2CARD), Flag-cGAS, Flag-STING, Flag-MDA5, Flag-MAVS, Flag-TBK1, Flag-IKKi, or Flag-IRF3 (5D) and increasing concentrations $(0,100$, or 200 ng) of plasmids, expressing TRIM9s or $200 \mathrm{ng}$ of TRIM9s-SA, TRIM9s-CA and TRIM9s- $\Delta$ RING. Empty pcDNA3.1 vector was used to maintain equal amounts of DNA among wells. Cells were collected at $24 \mathrm{~h}$ after transfection and luciferase activity was measured with the Dual-Luciferase Assay (Promega) with a Luminoskan Ascent luminometer (Thermo Scientific), according to the manufacturer's protocol. Reporter gene activity was determined by normalization of the firefly luciferase activity to renilla luciferase activity.

\section{Real-time PCR}

Total RNA was extracted using TRIzol reagent (Invitrogen) and reverse-transcribed using oligo-dT primers and reverse transcriptase (TAKARA). Real-time quantitative PCR was performed using SYBR green qPCR Mix kit (Genstar) and specific primers using the Primer 5.0 analyzer (Applied Biosystems). Data were normalized to the RPL13A gene, and the relative abundance of transcripts was calculated by the $2^{-\Delta \Delta C t}$ models. The following primers were used for real-time PCR:

TRIM9: Forward 5'-GTGTGCTACCAGTGCTTGGA-3'

Reverse 5'-TGTCTGACAGTCCGTTCAGC-3'

TRIM9s: Forward 5'-TGATGGCAACGGTGGTCAAT-3'

Reverse 5'-TGAAGGGCCTTACCCTCAGA-3'

TRIM9l: Forward 5'-CCTGATCCTGCCTTTGGTGT-3'

Reverse 5'-CCCCTTTTGTGATCCCTCCC-3'

IFN $\alpha-8$ : Forward 5'-TGATACTCCTGGCACAAAT-3'

Reverse 5'-TTGAGCCTTCTGGAACTGT-3'

IFN $\beta$ : Forward 5-CCTACAAAGAAGCAGCAA-3'

Reverse 5'-TCCTCAGGGATGTCAAAG-3'

TNF $\alpha$ : Forward 5'-CCAGACCAAGGTCAACCTCC-3'

Reverse 5'-CAGACTCGGCAAAGTCGAGA-3'

IL-6: Forward 5'-AGAGGCACTGGCAGAAAACAAC-3'

Reverse 5'-AGGCAAGTCTCCTCATTGAATCC-3'

ISG54: Forward 5'-GGAGGGAGAAAACTCCTTGGA-3'

Reverse 5'-GGCCAGTAGGTTGCACATTGT-3'

RPL13A: Forward 5'-GCCATCGTGGCTAAACAGGTA-3'

Reverse 5'-GTTGGTGTTCATCCGCTTGC-3'

GAPDH: Forward 5'-TCAAGAAGGTGGTGAAGCAG-3'

Reverse 5'-GAGGGGAGATTCAGTGTGGT-3'

$V S V$ gRNA: Forward 5'-TGCAAGGAAAGCATTGAACAA-3'

Reverse 5'-GAGGAGTCACCTGGACAATCACT-3'

HSV-1 glycoprotein C mRNA: Forward 5'-GCCAGATCGA CACGCAGACG-3'

Reverse 5'-CGAAATGGGCAGGGTGGACC-3'

\section{Immunoprecipitation and immunoblot analysis}

For immunoprecipitation, whole cell extracts were prepared after transfection or stimulation with appropriate ligands, followed by incubation overnight with the appropriate antibodies plus anti-Flag, anti-HA beads (Sigma), or Protein A/G beads (Pierce). Beads were washed five times with low-salt lysis buffer, and immunoprecipitates were eluted with $3 \times$ SDS Loading Buffer (Cell Signaling Technology) and resolved by SDS-PAGE. Proteins were transferred to PVDF membranes (Bio-Rad) and further incubated with the appropriate antibodies. LumiGlo Chemiluminescent Substrate System (KPL) was used for protein detection.

\section{RNA interference}

LipoRNAiMAX (Invitrogen) was used for transfection of siRNAs into 293T, A549 cells and PBMCs, according to the manufacturer's instructions. The sequences of siRNAs are as follows:

Human TRIM9-specific siRNAs:

TRIM9-siRNA-1\#:

SenseSeq: 5'-GCGCUACGUUGUCCUGGAA-3'

AntiSeq: 5'-UUCCAGGACAACGUAGCGC-3'

TRIM9-siRNA-2\#:

SenseSeq: 5'-GGCGCUGAACGGACUGUCA-3' AntiSeq: 5'-UGACAGUCCGUUCAGCGCC-3' 
TRIM9-siRNA-3\#:

SenseSeq: 5'-GCGUCAACAAGGAGCAUGA-3' AntiSeq: 5'-UCAUGCUCCUUGUUGACGC-3' Human TRIM9s or TRIM9l-specific siRNAs:

TRIM9S-siRNA-1\#:

SenseSeq: 5'-GCAGUAUCCCUCAGAGCGA-3' AntiSeq: 5'-UCGCUCUGAGGGAUACUGC-3' TRIM9s-siRNA-2\#:

SenseSeq: 5'-GCGAGAACUGCGUGGCAUC-3' AntiSeq: 5'-GAUGCCACGCAGUUCUCGC-3' TRIM9l-siRNA-1\#:

SenseSeq: 5'-CCACCCUGAUCCUGCCUUU-3' AntiSeq: 5'-AAAGGCAGGAUCAGGGUGG-3' TRIM9l-siRNA-2\#:

SenseSeq: 5'-GCAAUGUAUGUGGACAAUA-3'

AntiSeq: 5'-UAUUGUCCACAUACAUUGC-3'

Human $G S K 3 \beta$-specific siRNAs:

GSK3 $\beta$-siRNA-1\#:

SenseSeq: 5'-CAAAUGUCAAACUACCAAA-3' AntiSeq: 5'-UUUGGUAGUUUGACAUUUG-3' GSK3 $\beta$-siRNA-2\#:

SenseSeq: 5'-GCUAGAUCACUGUAACAUA-3' AntiSeq: 5'-UAUGUUACAGUGAUCUAGC-3' GSK3 $\beta$-siRNA-3\#:

SenseSeq: 5'-GGAAACAGUAUACAGAGUU-3'

AntiSeq: 5'-AACUCUGUAUACUGUUUCC-3'

GSK3 $\beta$-siRNA-4\#:

SenseSeq: 5'-CCCUCAAAUUAAGGCACAU-3'

AntiSeq: 5'-AUGUGCCUUAAUUUGAGGG-3'

Generation of TRIM9s KO cells by the CRISPR/Cas9 technology

TRIM9s KO A549 and 293T cells were generated by the CRIS$\mathrm{PR} / \mathrm{Cas} 9$ system, and the sequences of target sgRNA are as follows:

TRIM9s-sgRNA:

Forward 5'-CCCTCAGAGCGAGAACTGCG-3'

Reverse 5'-CGCAGTTCTCGCTCTGAGGG-3'

\section{Statistical analysis}

Data are represented as mean $\pm \mathrm{SD}$ when indicated, and Student's $t$-test was used for all statistical analyses with the GraphPad Prism 5.0 software. Differences between groups were considered significant when $P$-value was $<0.05$.

\section{Acknowledgments}

This work was supported by the National Basic Research Program of China (973 Program; 2015CB859800 and 2014CB910800), the National Natural Science Foundation of China (31370869 and 31522018), the Guangdong Natural Science Funds for Distinguished Young Scholar (S2013050014772), the Guangdong Innovative Research Team Program (2011Y035 and 201001Y0104687244), the Fundamental Research Funds for the Central Universities (15lgjc02), and NCI, NIH (CA090327, CA101795 and CA121191 to RFW).

\section{Author Contributions}

YQ, JC and RFW designed the study, interpreted data and wrote the manuscript; YQ, QL, ST and WX performed experiments and analyzed data.

\section{Competing Financial Interests}

The authors declare no competing financial interests.

\section{References}

1 Akira S, Uematsu S, Takeuchi O. Pathogen recognition and innate immunity. Cell 2006; 124:783-801.

2 Collins SE, Mossman KL. Danger, diversity and priming in innate antiviral immunity. Cytokine Growth Factor Rev 2014; 25:525-531.

3 O'Neill LA, Bowie AG. The family of five: TIR-domain-containing adaptors in Toll-like receptor signalling. Nat Rev Immunol 2007; 7:353-364.

4 Yoneyama M, Fujita T. RNA recognition and signal transduction by RIG-I-like receptors. Immunol Rev 2009; 227:54-65.

5 Chen G, Shaw MH, Kim YG, Nunez G. NOD-Like receptors: role in innate immunity and inflammatory disease. Annu Rev Pathol 2009; 4:365-398.

6 Barber GN. Innate immune DNA sensing pathways: STING, AIMII and the regulation of interferon production and inflammatory responses. Curr Opin Immunol 2011; 23:10-20.

7 Kato H, Takeuchi O, Sato S, et al. Differential roles of MDA5 and RIG-I helicases in the recognition of RNA viruses. Nature 2006; 441:101-105.

8 Seth RB, Sun L, Ea CK, Chen ZJ. Identification and characterization of MAVS, a mitochondrial antiviral signaling protein that activates NF-kappa B and IRF3. Cell 2005; 122:669682.

9 Zhang Z, Yuan B, Bao M, Lu N, Kim T, Liu YJ. The helicase DDX41 senses intracellular DNA mediated by the adaptor STING in dendritic cells. Nat Immunol 2011; 12:959-965.

10 Jakobsen MR, Bak RO, Andersen A, et al. IFI16 senses DNA forms of the lentiviral replication cycle and controls HIV-1 replication. Proc Natl Acad Sci USA 2013; 110:E4571-E4580.

11 Sun L, Wu J, Du F, Chen X, and Chen ZJ. Cyclic GMP-AMP synthase is a cytosolic DNA sensor that activates the type I interferon pathway. Science 2013; 339:786-791.

12 Fitzgerald KA, McWhirter SM, Faia KL, et al. IKK epsilon and TBK1 are essential components of the IRF3 signaling pathway. Nat Immunol 2003; 4:491-496.

13 Li S, Wang L, Berman M, Kong YY, Dorf ME. Mapping a dynamic innate immunity protein interaction network regulating type I interferon production. Immunity 2011; 35:426-440.

14 Wang C, Chen T, Zhang J, et al. The E3 ubiquitin ligase Nrdp1 "preferentially" promotes TLR-mediated production of type I interferon. Nat Immunol 2009; 10:744-752.

15 Cui J, Li Y, Zhu L, et al. NLRP4 negatively regulates type I interferon signaling by targeting the kinase TBK1 for degradation via the ubiquitin ligase DTX4. Nat Immunol 2012; 13:387-395.

16 Zhang M, Wang L, Zhao X, et al. TRAF-interacting protein (TRIP) negatively regulates IFN-beta production and antiviral response by promoting proteasomal degradation of TANK-binding kinase 1. J Exp Med 2012; 209:1703-1711.

17 Zhao W. Negative regulation of TBK1-mediated antiviral immunity. FEBS Lett 2013; 587:542-548. 
18 Lei CQ, Zhong B, Zhang Y, Zhang J, Wang S, Shu HB. Glycogen synthase kinase 3 beta regulates irf3 transcription factor-mediated antiviral response via activation of the kinase TBK1. Immunity 2010; 33:878-889.

19 Rajsbaum R, Garcia-Sastre A, Versteeg GA. TRIMmunity: the roles of the TRIM E3-ubiquitin ligase family in innate antiviral immunity. J Mol Biol 2014; 426:1265-1284.

20 Yan J, Li Q, Mao AP, Hu MM, Shu HB. TRIM4 modulates type I interferon induction and cellular antiviral response by targeting RIG-I for K63-linked ubiquitination. J Mol Cell Biol 2014; 6:154-163.

21 Gack MU, Shin YC, Joo CH, et al. TRIM25 RING-finger E3 ubiquitin ligase is essential for RIG-I-mediated antiviral activity. Nature 2007; 446:916-920.

22 Zhang J, Hu MM, Wang YY, Shu HB. TRIM32 protein modulates type $\mathrm{i}$ interferon induction and cellular antiviral response by targeting MITA/STING protein for K63-linked Ubiquitination. J Biol Chem 2012; 287:28646-28655.

23 Tsuchida T, Zou J, Saitoh T, et al. The Ubiquitin Ligase TRIM56 Regulates Innate Immune Responses to Intracellular Double-Stranded DNA. Immunity 2010; 33:765-776.

24 Zhang Z, Bao M, Lu N, Wemg L, Yuan B, Liu YJ. The E3 ubiquitin ligase TRIM21 negatively regulates the innate immune response to intracellular double-stranded DNA. Nat Immunol 2013; 14:172-178.

25 Versteeg GA, Rajsbaum R, Sanchez-Aparicio MT, et al. The E3-ligase TRIM family of proteins regulates signaling pathways triggered by innate immune pattern-recognition receptors. Immunity 2013; 38:384-398.

26 Tanji K, Kamitani T, Mori F, Kakita A, Takahashi H, Wakabayashi K. TRIM9, a novel brain-specific E3 ubiquitin ligase, is repressed in the brain of Parkinson's disease and dementia with Lewy bodies. Neurobiol Dis 2010; 38:210-218.

27 Berti C, Messali S, Ballabio A, Reymond A, Meroni G. TRIM9 is specifically expressed in the embryonic and adult nervous system. Mech Dev 2002; 113:159-162.

28 Shi M, Cho H, Inn KS, et al. Negative regulation of NF-kappa B activity by brain-specific TRIpartite Motif protein 9. Nat Commun 2014; 5:4820.

29 Toniato E, Chen XP, Losman J, Flati V, Donahue L, Rothman P. TRIM8/GERP RING finger protein interacts with SOCS-1. J Biol Chem 2002; 277:37315-37322.

30 Zhou Z, Jia X, Xue Q, et al. TRIM14 is a mitochondrial adaptor that facilitates retinoic acid-inducible gene-I-like receptor-mediated innate immune response. Proc Natl Acad Sci USA 2014; 111:E245-E254.
31 Yang K, Shi HX, Liu XY, et al. TRIM21 is essential to sustain IFN regulatory factor 3 activation during antiviral response. $J$ Immunol 2009; 182:3782-3792.

32 Lee Y, Song B, Park C, Kwon KS. TRIM11 negatively regulates IFNb production and antiviral activity by targeting TBK1. PLoS One 2013; 8:e63255.

33 Xue Q, Zhou Z, Lei X, et al. TRIM38 negatively regulates TLR3-mediated IFN-b signaling by targeting TRIF for degradation. PLoS One 2012; 7:e46825.

34 Carthagena L, Bergamaschi A, Luna JM, et al. Human TRIM gene expression in response to interferons. PLoS One 2009; 4:e4894.

35 Shalem O, Sanjana NE, Hartenian E, et al. Genome-scale CRISPR-Cas9 knockout screening in human cells. Science 2014; 343:84-87.

36 Versteeg GA, Benke S, Garcia-Sastre A, Rajsbaum R. InTRIMsic immunity: positive and negative regulation of immune signaling by tripartite motif proteins. Cytokine Growth Factor Rev 2014; 25:563-576.

37 Shi M, Deng W, Bi E, et al. TRIM30 alpha negatively regulates TLR-mediated NF-kappa B activation by targeting TAB2 and TAB3 for degradation. Nat Immunol 2008; 9:369377.

38 Zhao W, Wang L, Zhang M, et al. Tripartite motif-containing protein 38 negatively regulates TLR3/4- and RIG-I-mediated IFN- $\beta$ production and antiviral response by targeting NAP1. $J$ Immunol 2012; 188:5311-5318.

39 Arimoto K, Funami K, Saeki Y, et al. Polyubiquitin conjugation to NEMO by triparite motif protein 23 (TRIM23) is critical in antiviral defense. Proc Natl Acad Sci USA 2010; 107:15856-15861.

40 Mandell MA, Jain A, Arko-Mensah J, et al. TRIM proteins regulate autophagy and can target autophagic substrates by direct recognition. Dev Cell 2014; 30:394-409.

41 Cui J, Zhu L, Xia X, et al. NLRC5 negatively regulates the NF-kappa B and Type I interferon signaling pathways. Cell 2010; 141:483-496.

42 Xia XJ, Cui J, Wang HY, et al. NLRX1 Negatively regulates TLR-induced NF-kappa B signaling by targeting TRAF6 and IKK. Immunity 2011; 34:843-853.

43 Lei Y, Wen H, Yu Y, et al. The mitochondrial proteins NLRX1 and TUFM form a complex that regulates type I interferon and autophagy. Immunity 2012; 36:933-946.

44 El Asmi F, Maroui MA, Dutrieux J, Blondel D, Nisole S, Chelbi-Alix MK. Implication of PMLIV in both intrinsic and innate immunity. Plos Pathog 2014; 10:e1003975.

(Supplementary information is linked to the online version of the paper on the Cell Research website.) 